\title{
Sampling theories of boundary value problems with several internal points of discontinuity
}

Mohammed M Tharwat ${ }^{*}$

${ }^{*}$ Correspondence:

zahraa26@yahoo.com Department of Mathematics, Faculty of Science, University of Jeddah, Jeddah, Saudi Arabia Department of Mathematics, Faculty of Science, Beni-Suef University, Beni-Suef, Egypt

\begin{abstract}
In this paper, our boundary value problem is a Dirac system with transmission conditions at several points of discontinuity. The main purpose of this paper is to derive the sampling theorems of this boundary value problem. To derive the sampling theorems including the construction of the Green's matrix as well as the vector-valued eigenfunction expansion theorem, we briefly study the spectral analysis of the problem as in Levitan and Sargsjan (Introduction to Spectral Theory: Selfadjoint Ordinary Differential Operators, Translations of Mathematical Monographs, vol. 39, 1975; Sturm-Liouville and Dirac Operators, 1991) in a way similar to that of Fulton (Proc. R. Soc. Edinb., Sect. A 77:293-308, 1977). We derive sampling representations for transforms whose kernels are either solutions or the Green's matrices of the problem. In the special case when our problem has one point of discontinuity, the obtained results coincide with the corresponding results in Tharwat et al. (Numer. Funct. Anal. Optim. 34:323-348, 2013).
\end{abstract}

MSC: 34L16; 94A20; 65L15

Keywords: sampling theory; Dirac systems; transmission conditions; discontinuous boundary value problems; Green's matrix

\section{Introduction}

Let $\mathfrak{H}(D)$ be a class of complex-valued functions defined on $D$, where $D$ is a subset of $\mathbb{C}$, which may coincide with $\mathbb{C}$. We say that a sampling theorem holds for the class $\mathfrak{H}(D)$ if there are two sequences $\left\{\lambda_{k}\right\}_{k=1}^{\infty} \subset D$ and $\left\{S_{k}(\lambda)\right\}_{k=1}^{\infty} \subset \mathfrak{H}(D)$, such that

$$
F(\lambda)=\sum_{k=1}^{\infty} F\left(\lambda_{k}\right) S_{k}(\lambda), \quad \lambda \in D, F \in \mathfrak{H}(D),
$$

where series (1.1) satisfies some sort of convergence. The points $\left\{\lambda_{k}\right\}_{k=1}^{\infty}$, which are for practical reasons preferred to be real, will be called the sampling points and the functions $\left\{S_{k}(\lambda)\right\}_{k=1}^{\infty}$ are called the sampling (reconstructing) functions. The name sampling comes about since the elements of $\left\{S_{k}(\lambda)\right\}_{k=1}^{\infty}$, when a sampling theorem holds, can be recovered from their values at $\left\{\lambda_{k}\right\}_{k=1}^{\infty}$. When a sampling theorem holds for a class $\left\{S_{k}(\lambda)\right\}_{k=1}^{\infty}$, this may have many applications when the discrete (digital) state is preferable, like the role

(c) 2016 Tharwat. This article is distributed under the terms of the Creative Commons Attribution 4.0 International License (http://creativecommons.org/licenses/by/4.0/), which permits unrestricted use, distribution, and reproduction in any medium, provided you give appropriate credit to the original author(s) and the source, provide a link to the Creative Commons license, and indicate if changes were made. 
played by the Whittaker-Shannon-Kotel'nikov (WSK) sampling theorem [5-9] in signal processing.

The point now concerns the classes $\mathfrak{H}(D)$, for which sampling theorems are guaranteed. In most of the known cases in sampling theory the classes of the sampled functions are integral transforms defined on a $L^{2}$-space. Now, in the following, we give an example.

Example 1.1 Let $\mathfrak{H}(\mathbb{C})$ be the space of all bandlimited functions, i.e., functions of the form of finite Fourier transforms,

$$
F(\lambda)=\frac{1}{\sqrt{2 \pi}} \int_{-\pi}^{\pi} f(x) e^{i x \lambda} d x, \quad f(\cdot) \in L^{2}(-\pi, \pi), \lambda \in \mathbb{C} .
$$

Thus, $\mathfrak{H}(\mathbb{C})$ is the Paley-Wiener space $P W_{\pi}^{2}$ of entire functions of exponential type at most $\pi$, which belong to $L^{2}(\mathbb{R})$ when restricted to $\mathbb{R}, c f$. [5, 10,11]. Elements of $\mathfrak{H}(\mathbb{C})$, in this case, have sampling expansions of the form (1.1) with

$$
\lambda_{k}=k ; \quad S_{k}(\lambda)=\frac{\sin \pi(\lambda-k)}{\pi(\lambda-k)}, \quad k \in \mathbb{Z},
$$

where the sampling expansions converge absolutely on $\mathbb{C}$ and uniformly on both $\mathbb{R}$ and compact subsets of $\mathbb{C}$, [12], p.262. This result is the well-known WSK sampling theorem [5-9] which has many applications in signal processing.

The above example is a special case of a generalized sampling theorem for generalized integral transforms. It is known as Kramer's sampling theorem [5, 13-16] and reads as follows.

Theorem 1.1 Let $\left\{\lambda_{k}\right\}_{k=-\infty}^{\infty}$ be a sequence of real numbers. Let $I \subseteq \mathbb{R}$ and $\mathcal{K}(\cdot, \lambda): I \times \mathbb{C} \rightarrow \mathbb{C}$ be a function such that

1. $\mathcal{K}(\cdot, \lambda) \in L^{2}(I)$ for all $\lambda \in \mathbb{C}$,

2. the sequence $\left\{\mathcal{K}\left(\cdot, \lambda_{k}\right)\right\}_{k=-\infty}^{\infty}$ forms a complete orthogonal set in $L^{2}(I)$.

Let $\mathfrak{H}(\mathbb{C})$ be the set of integral transforms

$$
F(\lambda)=\int_{I} f(x) \mathcal{K}(x, \lambda) d x, \quad f(\cdot) \in L^{2}(I)
$$

Then elements of $\mathfrak{H}(\mathbb{C})$ admit the sampling expansions

$$
F(\lambda)=\sum_{k=-\infty}^{\infty} F\left(\lambda_{k}\right) \frac{\int_{I} \mathcal{K}(x, \lambda) \overline{\mathcal{K}\left(x, \lambda_{k}\right)} d x}{\int_{I}\left|\mathcal{K}\left(x, \lambda_{k}\right)\right|^{2} d x}, \quad F \in \mathfrak{H}(\mathbb{C}) .
$$

Series (1.4) converges uniformly wherever $\|\mathcal{K}(\cdot, \lambda)\|_{L^{2}(I)}$ is bounded.

We can see that Example 1.1 above is a special case of Theorem 1.1. There are many problems motivated by Kramer's theorem. The first concerns the situation for which we can obtain the kernel and the sampling points such that the theorem holds. The second concerns the relationship between Kramer's sampling expansions and Lagrange-type interpolation ones. Notice that the sampling series in Example 1.1 is in the form of a Lagrange-type interpolation expansion. A partial answer to the first question was given by Weiss [17], 
before the existence of Kramer's theorem. In this short note [17], the kernel is extracted from the second order boundary value problem

$$
\begin{aligned}
& -y^{\prime \prime}(x)+q(x) y(x)=\lambda y(x), \quad-\infty<a \leq x \leq b<\infty, \\
& \cos \alpha y(a)+\sin \alpha y^{\prime}(a)=0, \\
& \cos \beta y(b)+\sin \beta y^{\prime}(b)=0,
\end{aligned}
$$

where $q(\cdot)$ is continuous and real valued on $[a, b]$ and $\alpha, \beta \in[0, \pi)$. If we take $u(\cdot, \lambda)$ to be the solution of (1.6) determined by the initial conditions

$$
u(a, \lambda)=\sin \alpha, \quad u^{\prime}(a, \lambda)=-\cos \alpha, \quad \lambda \in \mathbb{C},
$$

then $u(\cdot, \lambda)$ satisfies Kramer's theorem with respect to the sequence of the eigenvalues, i.e., the sequence of the eigenvalues, $\left\{\lambda_{k}\right\}_{k=0}^{\infty}$, will be taken as the sampling points. For the second question, it is shown in many articles that Kramer's expansions are nothing more than Lagrange-type interpolation ones when the kernels of the sampled integral transforms are solutions of certain self adjoint eigenvalue problems or are to be expressed in terms of the Green's functions of these problems; see [18-20]. Using the connection between Kramer's theorem and the eigenvalue problems most of the known sampling expansions can easily be obtained.

In [21,22], the authors discussed sampling theorems of regular one-dimensional continuous Dirac systems; see also [23]. In the case of boundary value problems with one point of discontinuity, Tharwat et al. [4], discussed sampling theorems of Dirac systems; see also $[24,25]$. In the present paper we derive sampling theorems associated with Dirac systems with transmission conditions at several points of discontinuity. That is, we define classes of transforms associated with these problems and derive sampling expansions for these classes. We use both solutions and the Green's matrices to define the sampled transforms. In all cases the obtained sampling expansions are written in the form of a Lagrange interpolation series. The uniform convergence of the obtained sampling expansion as well as the analytic and growth properties of the sampled transforms are given. However, sampling theories associated with Dirac systems, which have several points of discontinuity, do not exist as far as we know. An illustrative example is given in detail in the last section.

\section{Preliminaries}

In this section we introduce some of the notations and relations that will be used in the sequel; then we prove some useful lemmas and theorems. We consider the Dirac system

$$
\begin{aligned}
& \left.\begin{array}{l}
r(x) u_{2}^{\prime}(x)-q_{1}(x) u_{1}(x)=\lambda u_{1}(x), \\
r(x) u_{1}^{\prime}(x)+q_{2}(x) u_{2}(x)=-\lambda u_{2}(x),
\end{array}\right\} \quad x \in\left[a, c_{1}\right) \cup\left(c_{1}, c_{2}\right) \cup\left(c_{2}, c_{3}\right) \cup \cdots \cup\left(c_{m}, b\right], \\
& \mathcal{U}_{1}(u):=\sin \alpha u_{1}(a)+\cos \alpha u_{2}(a)=0, \\
& \mathcal{U}_{2}(u):=\sin \beta u_{1}(b)+\cos \beta u_{2}(b)=0,
\end{aligned}
$$

and the transmission conditions

$$
\mathcal{L}_{k}(u):=\left[\begin{array}{l}
\gamma_{k} u_{1}\left(c_{k}^{-}\right) \\
\gamma_{k}^{\prime} u_{2}\left(c_{k}^{-}\right)
\end{array}\right]-\left[\begin{array}{l}
\delta_{k} u_{1}\left(c_{k}^{+}\right) \\
\delta_{k}^{\prime} u_{2}\left(c_{k}^{+}\right)
\end{array}\right]=0, \quad k=1,2, \ldots, m,
$$


where $\lambda \in \mathbb{C}$;

$$
r(x):= \begin{cases}r_{1}, & x \in\left[a, c_{1}\right) \\ r_{k}, & x \in\left(c_{k-1}, c_{k}\right)(k=2,3, \ldots, m) \\ r_{m+1}, & x \in\left(c_{m}, b\right]\end{cases}
$$

$r_{k}>0(k=1,2, \ldots, m+1)$ are real numbers; the real-valued functions $q_{1}(\cdot)$ and $q_{2}(\cdot)$ are continuous in $\left[a, c_{1}\right),\left(c_{k-1}, c_{k}\right)$, and $\left(c_{m}, b\right](k=2,3, \ldots, m)$, and have finite limits $q_{1}\left(c_{k}^{ \pm}\right):=$ $\lim _{x \rightarrow c_{k}^{ \pm}} q_{1}(x), q_{2}\left(c_{k}^{ \pm}\right):=\lim _{x \rightarrow c_{k}^{ \pm}} q_{2}(x)(k=1,2, \ldots, m) ; \gamma_{k}, \delta_{k}, \gamma_{k}^{\prime}, \delta_{k}^{\prime} \in \mathbb{R}, \gamma_{k}, \delta_{k}, \gamma_{k}^{\prime}, \delta_{k}^{\prime} \neq 0$ and $\alpha, \beta \in[0, \pi)$; see $[26,27]$.

Let $\mathcal{H}$ be the Hilbert space

$$
\mathcal{H}:=\left\{u(x)=\left(\begin{array}{l}
u_{1}(x) \\
u_{2}(x)
\end{array}\right), u_{1}(x), u_{2}(x) \in \bigoplus_{k=1}^{m+1} L^{2}\left(c_{k-1}, c_{k}\right), a=c_{0}, b=c_{m+1}\right\} .
$$

The inner product of $\mathcal{H}$ is defined by

$$
\begin{aligned}
\langle u(\cdot), v(\cdot)\rangle_{\mathcal{H}}:= & \frac{1}{r_{1}} \int_{a}^{c_{1}} u^{\top}(x) \bar{v}(x) d x+\sum_{k=2}^{m} \frac{\prod_{i=1}^{k-1} D_{i}}{r_{k}} \int_{c_{k-1}}^{c_{k}} u^{\top}(x) \bar{v}(x) d x \\
& +\frac{\prod_{i=1}^{m} D_{i}}{r_{m+1}} \int_{c_{m}}^{b} u^{\top}(x) \bar{v}(x) d x,
\end{aligned}
$$

where $D_{i}=\frac{\delta_{i} \delta_{i}^{\prime}}{\gamma_{i} \gamma_{i}^{\prime}}, D_{i}>0, i=1,2, \ldots, m$, and $\top$ denotes the matrix transpose,

$$
u(x)=\left(\begin{array}{l}
u_{1}(x) \\
u_{2}(x)
\end{array}\right), v(x)=\left(\begin{array}{l}
v_{1}(x) \\
v_{2}(x)
\end{array}\right) \in \mathcal{H}, \quad u_{i}(\cdot), v_{i}(\cdot) \in \bigoplus_{k=1}^{m+1} L^{2}\left(c_{k-1}, c_{k}\right), \quad i=1,2 .
$$

For vector-valued functions $u(x)$, which are defined on $\left[a, c_{1}\right) \cup\left(c_{1}, c_{2}\right) \cup\left(c_{2}, c_{3}\right) \cup \cdots \cup$ $\left(c_{m}, b\right]$ and have a finite limit $u\left(c_{k}^{ \pm}\right):=\lim _{x \rightarrow c_{k}^{ \pm}} u(x)(k=1,2, \ldots, m)$, by $u_{(k)}(x)(k=$ $1,2, \ldots, m+1)$ we denote the functions

$$
\begin{aligned}
& u_{(1)}(x)=\left\{\begin{array}{ll}
u(x), & x \in\left[a, c_{1}\right), \\
u\left(c_{1}^{-}\right), & x=c_{1},
\end{array} u_{(i)}(x)= \begin{cases}u\left(c_{i-1}^{+}\right), & x=c_{i-1}, \\
u(x), & x \in\left(c_{i-1}, c_{i}\right), \quad i=2,3, \ldots, m, \\
u\left(c_{i}^{-}\right), & x=c_{i},\end{cases} \right. \\
& u_{(m+1)}(x)= \begin{cases}u(x), & x \in\left(c_{m}, b\right], \\
u\left(c_{m}^{+}\right), & x=c_{m},\end{cases}
\end{aligned}
$$

which are defined on $\mathcal{I}_{1}:=\left[a, c_{1}\right], \mathcal{I}_{k}:=\left[c_{k-1}, c_{k}\right](k=2,3, \ldots, m)$ and $\mathcal{I}_{m+1}:=\left[c_{m}, b\right]$, respectively.

In the following lemma, we will prove that the eigenvalues of the problem (2.1)-(2.4) are real.

Lemma 2.1 The eigenvalues of the problem (2.1)-(2.4) are real.

Proof Assume to the contrary that $\lambda_{0}$ is a non-real eigenvalue of problem (2.1)-(2.4). Let $\left(\begin{array}{l}u_{1}(x) \\ u_{2}(x)\end{array}\right)$ be a corresponding (non-trivial) vector-valued eigenfunction. By (2.1), we 
have

$$
\begin{aligned}
& r(x) \frac{d}{d x}\left\{u_{1}(x) \bar{u}_{2}(x)-\bar{u}_{1}(x) u_{2}(x)\right\} \\
& \quad=\left(\bar{\lambda}_{0}-\lambda_{0}\right)\left\{\left|u_{1}(x)\right|^{2}+\left|u_{2}(x)\right|^{2}\right\}, \quad x \in\left[a, c_{1}\right) \cup\left(c_{1}, c_{2}\right) \cup\left(c_{2}, c_{3}\right) \cup \cdots \cup\left(c_{m}, b\right] .
\end{aligned}
$$

Integrating the above equation through $\left[a, c_{1}\right),\left(c_{k-1}, c_{k}\right)(k=2,3, \ldots, m)$, and $\left(c_{m}, b\right]$, we obtain

$$
\begin{aligned}
& \frac{\bar{\lambda}_{0}-\lambda_{0}}{r_{1}}\left[\int_{a}^{c_{1}}\left(\left|u_{1}(x)\right|^{2}+\left|u_{2}(x)\right|^{2}\right) d x\right] \\
& \quad=u_{1}\left(c_{1}^{-}\right) \bar{u}_{2}\left(c_{1}^{-}\right)-\bar{u}_{1}\left(c_{1}^{-}\right) u_{2}\left(c_{1}^{-}\right)-\left[u_{1}(a) \bar{u}_{2}(a)-\bar{u}_{1}(a) u_{2}(a)\right], \\
& \frac{\bar{\lambda}_{0}-\lambda_{0}}{r_{k}}\left[\int_{c_{k-1}}^{c_{k}}\left(\left|u_{1}(x)\right|^{2}+\left|u_{2}(x)\right|^{2}\right) d x\right] \\
& =u_{1}\left(c_{k}^{-}\right) \bar{u}_{2}\left(c_{k}^{-}\right)-\bar{u}_{1}\left(c_{k}^{-}\right) u_{2}\left(c_{k}^{-}\right)-\left[u_{1}\left(c_{k-1}^{+}\right) \bar{u}_{2}\left(c_{k-1}^{+}\right)-\bar{u}_{1}\left(c_{k-1}^{+}\right) u_{2}\left(c_{k-1}^{+}\right)\right],
\end{aligned}
$$

for $k=2,3, \ldots, m$, and

$$
\begin{aligned}
& \frac{\bar{\lambda}_{0}-\lambda_{0}}{r_{m+1}}\left[\int_{c_{m}}^{b}\left(\left|u_{1}(x)\right|^{2}+\left|u_{2}(x)\right|^{2}\right) d x\right] \\
& \quad=u_{1}(b) \bar{u}_{2}(b)-\bar{u}_{1}(b) u_{2}(b)-\left[u_{1}\left(c_{m}^{+}\right) \bar{u}_{2}\left(c_{m}^{+}\right)-\bar{u}_{1}\left(c_{m}^{+}\right) u_{2}\left(c_{m}^{+}\right)\right] .
\end{aligned}
$$

Then from (2.2), (2.3), and the transmission conditions, we have, respectively,

$$
\begin{aligned}
& u_{1}(a) \bar{u}_{2}(a)-\bar{u}_{1}(a) u_{2}(a)=0, \\
& u_{1}(b) \bar{u}_{2}(b)-\bar{u}_{1}(b) u_{2}(b)=0,
\end{aligned}
$$

and

$$
\begin{aligned}
& u_{1}\left(c_{k}^{-}\right) \bar{u}_{2}\left(c_{k}^{-}\right)-\bar{u}_{1}\left(c_{k}^{-}\right) u_{2}\left(c_{k}^{-}\right) \\
& \quad=\frac{\delta_{k} \delta_{k}^{\prime}}{\gamma_{k} \gamma_{k}^{\prime}}\left[u_{1}\left(c_{k}^{+}\right) \bar{u}_{2}\left(c_{k}^{+}\right)-\bar{u}_{1}\left(c_{k}^{+}\right) u_{2}\left(c_{k}^{+}\right)\right], \quad k=1,2, \ldots, m .
\end{aligned}
$$

Since $\lambda_{0} \neq \bar{\lambda}_{0}$, it follows from the last three equations and (2.8), (2.9), (2.10) that

$$
\begin{aligned}
& \frac{1}{r_{1}} \int_{a}^{c_{1}}\left(\left|u_{1}(x)\right|^{2}+\left|u_{2}(x)\right|^{2}\right) d x+\sum_{k=2}^{m} \frac{\prod_{i=1}^{k-1} D_{i}}{r_{k}} \int_{c_{k-1}}^{c_{k}}\left(\left|u_{1}(x)\right|^{2}+\left|u_{2}(x)\right|^{2}\right) d x \\
& \quad+\frac{\prod_{i=1}^{m} D_{i}}{r_{m+1}} \int_{c_{m}}^{b}\left(\left|u_{1}(x)\right|^{2}+\left|u_{2}(x)\right|^{2}\right) d x=0 .
\end{aligned}
$$

Then $u_{i}(x)=0, i=1,2$, and this is a contradiction. Consequently, $\lambda_{0}$ must be real.

Lemma 2.2 Let $\lambda_{1}$ and $\lambda_{2}$ be two different eigenvalues of the problem (2.1)-(2.4). Then the corresponding vector-valued eigenfunctions $u\left(x, \lambda_{1}\right)$ and $v\left(x, \lambda_{2}\right)$ of this problem satisfy the 
following equality:

$$
\begin{aligned}
& \frac{1}{r_{1}} \int_{a}^{c_{1}} u^{\top}\left(x, \lambda_{1}\right) v\left(x, \lambda_{2}\right) d x+\sum_{k=2}^{m} \frac{\prod_{i=1}^{k-1} D_{i}}{r_{k}} \int_{c_{k-1}}^{c_{k}} u^{\top}\left(x, \lambda_{1}\right) v\left(x, \lambda_{2}\right) d x \\
& \quad+\frac{\prod_{i=1}^{m} D_{i}}{r_{m+1}} \int_{c_{m}}^{b} u^{\top}\left(x, \lambda_{1}\right) v\left(x, \lambda_{2}\right) d x=0 .
\end{aligned}
$$

Proof By (2.1) we obtain

$$
\begin{aligned}
& r(x) \frac{d}{d x}\left\{u_{1}\left(x, \lambda_{1}\right) v_{2}\left(x, \lambda_{2}\right)-u_{2}\left(x, \lambda_{2}\right) v_{1}\left(x, \lambda_{1}\right)\right\} \\
& \quad=\left(\lambda_{2}-\lambda_{1}\right)\left\{u_{1}\left(x, \lambda_{1}\right) v_{1}\left(x, \lambda_{2}\right)+u_{2}\left(x, \lambda_{1}\right) v_{2}\left(x, \lambda_{2}\right)\right\} .
\end{aligned}
$$

Integrating the above equation through $\left[a, c_{1}\right),\left(c_{k-1}, c_{k}\right)(k=2,3, \ldots, m)$, and $\left(c_{m}, b\right]$, and taking into account that $u\left(x, \lambda_{1}\right)$ and $v\left(x, \lambda_{2}\right)$ satisfy (2.2)-(2.4), we obtain (2.12), where $\lambda_{1} \neq \lambda_{2}$.

Now we will construct a special fundamental system of solutions of (2.1) for $\lambda$ not being an eigenvalue. By virtue of Theorem 1.1 in [1], we will define the two solutions of (2.1)

$$
\varphi(\cdot, \lambda)=\left(\begin{array}{l}
\varphi_{1}(\cdot, \lambda) \\
\varphi_{2}(\cdot, \lambda)
\end{array}\right), \quad \chi(\cdot, \lambda)=\left(\begin{array}{l}
\chi_{1}(\cdot, \lambda) \\
\chi_{2}(\cdot, \lambda)
\end{array}\right),
$$

where

$$
\begin{aligned}
& \varphi_{1}(x, \lambda)= \begin{cases}\varphi_{1,1}(x, \lambda), & x \in\left[c_{0}, c_{1}\right), \\
\varphi_{1,2}(x, \lambda), & x \in\left(c_{1}, c_{2}\right), \\
\ldots, & \\
\varphi_{1, m+1}(x, \lambda), & x \in\left(c_{m}, c_{m+1}\right],\end{cases} \\
& \varphi_{2}(x, \lambda)= \begin{cases}\varphi_{2,1}(x, \lambda), & x \in\left[c_{0}, c_{1}\right), \\
\varphi_{2,2}(x, \lambda), & x \in\left(c_{1}, c_{2}\right), \\
\ldots, & \\
\varphi_{2, m+1}(x, \lambda), & x \in\left(c_{m}, c_{m+1}\right],\end{cases} \\
& \chi_{1}(x, \lambda)= \begin{cases}\chi_{1,1}(x, \lambda), & x \in\left[c_{0}, c_{1}\right), \\
\chi_{1,2}(x, \lambda), & x \in\left(c_{1}, c_{2}\right), \\
\ldots, & \\
\chi_{1, m+1}(x, \lambda), & x \in\left(c_{m}, c_{m+1}\right],\end{cases} \\
& \chi_{2}(x, \lambda)= \begin{cases}\chi_{2,1}(x, \lambda), & x \in\left[c_{0}, c_{1}\right), \\
\chi_{2,2}(x, \lambda), & x \in\left(c_{1}, c_{2}\right), \\
\ldots, & \\
\chi_{2, m+1}(x, \lambda), & x \in\left(c_{m}, c_{m+1}\right],\end{cases}
\end{aligned}
$$

as follows, where $c_{0}=a$ and $c_{m+1}=b$. By virtue of Theorem 1.1 in [1] the initial-value problem

$$
\begin{aligned}
& r(x) u_{2}^{\prime}(x)-q_{1}(x) u_{1}(x)=\lambda u_{1}(x), \\
& r(x) u_{1}^{\prime}(x)+q_{2}(x) u_{2}(x)=-\lambda u_{2}(x), \quad x \in\left(a, c_{1}\right),
\end{aligned}
$$




$$
u_{1}(a)=\cos \alpha, \quad u_{2}(a)=-\sin \alpha
$$

has a unique solution $u=\left(\begin{array}{l}\varphi_{1,1}(x, \lambda) \\ \varphi_{2,1}(x, \lambda)\end{array}\right)$, which is an entire function of $\lambda \in \mathbb{C}$ for each fixed $x \in\left[a, c_{1}\right]$. Similarly, employing the same method as in proof of Theorem 1.1 in [1], we see that the problem

$$
\begin{aligned}
& r(x) u_{2}^{\prime}(x)-q_{1}(x) u_{1}(x)=\lambda u_{1}(x), \\
& r(x) u_{1}^{\prime}(x)+q_{2}(x) u_{2}(x)=-\lambda u_{2}(x), \quad x \in\left(c_{m}, b\right), \\
& u_{1}(b)=\cos \beta, \quad u_{2}(b)=-\sin \beta,
\end{aligned}
$$

has a unique solution $u=\left(\begin{array}{l}\chi_{1, m+1}(x, \lambda) \\ \chi_{2, m+1}(x, \lambda)\end{array}\right)$, which is an entire function of the parameter $\lambda$ for each fixed $x \in\left[c_{m}, b\right]$.

Now the functions $\varphi_{i, k+1}(x, \lambda)$ and $\chi_{i, k}(x, \lambda)$ are defined in terms of $\varphi_{i, k}(x, \lambda)$ and $\chi_{i, k+1}(x, \lambda), i=1,2, k=1,2, \ldots, m$, respectively, as follows. The initial-value problem

$$
\begin{aligned}
& r(x) u_{2}^{\prime}(x)-q_{1}(x) u_{1}(x)=\lambda u_{1}(x), \\
& r(x) u_{1}^{\prime}(x)+q_{2}(x) u_{2}(x)=-\lambda u_{2}(x), \quad x \in\left(c_{k}, c_{k+1}\right), \\
& u_{1}\left(c_{k}\right)=\frac{\gamma_{k}}{\delta_{k}} \varphi_{1, k}\left(c_{k}^{-}, \lambda\right), \quad u_{2}\left(c_{k}\right)=\frac{\gamma_{k}^{\prime}}{\delta_{k}^{\prime}} \varphi_{2, k}\left(c_{k}^{-}, \lambda\right), \quad k=1,2, \ldots, m,
\end{aligned}
$$

has a unique solution $u=\left(\begin{array}{c}\varphi_{1, k+1}(x, \lambda) \\ \varphi_{2, k+1}(x, \lambda)\end{array}\right)$ for each $\lambda \in \mathbb{C}$.

Similarly, the following problem also has a unique solution $u=\left(\begin{array}{l}\chi_{1, k}(x, \lambda) \\ \chi_{2, k}(x, \lambda)\end{array}\right)$ :

$$
\begin{aligned}
& r(x) u_{2}^{\prime}(x)-q_{1}(x) u_{1}(x)=\lambda u_{1}(x), \\
& r(x) u_{1}^{\prime}(x)+q_{2}(x) u_{2}(x)=-\lambda u_{2}(x), \quad x \in\left(c_{k-1}, c_{k}\right), \\
& u_{1}\left(c_{k}\right)=\frac{\delta_{k}}{\gamma_{k}} \chi_{1, k+1}\left(c_{k}^{+}, \lambda\right), \quad u_{2}\left(c_{k}\right)=\frac{\delta_{k}^{\prime}}{\gamma_{k}^{\prime}} \chi_{2, k+1}\left(c_{k}^{+}, \lambda\right), \quad k=1,2, \ldots, m .
\end{aligned}
$$

By virtue of equations (2.20) and (2.22) these solutions satisfy both transmission conditions (2.4). These functions are entire in $\lambda$ for all $x \in\left[a, c_{1}\right) \cup\left(c_{1}, c_{2}\right) \cup\left(c_{2}, c_{3}\right) \cup \cdots \cup\left(c_{m}, b\right]$.

Let $W(\varphi, \chi)(\cdot, \lambda)$ denote the Wronskian of $\varphi(\cdot, \lambda)$ and $\chi(\cdot, \lambda)$ defined in [2], p.194, i.e.,

$$
W(\varphi, \chi)(\cdot, \lambda):=\left|\begin{array}{ll}
\varphi_{1}(\cdot, \lambda) & \varphi_{2}(\cdot, \lambda) \\
\chi_{1}(\cdot, \lambda) & \chi_{2}(\cdot, \lambda)
\end{array}\right| .
$$

It is obvious that the Wronskians

$$
\begin{aligned}
\omega_{k}(\lambda) & :=W(\varphi, \chi)(x, \lambda) \\
& =\varphi_{1, k}(x, \lambda) \chi_{2, k}(x, \lambda)-\varphi_{2, k}(x, \lambda) \chi_{1, k}(x, \lambda), \quad x \in \mathcal{I}_{k}, k=1,2, \ldots, m+1
\end{aligned}
$$

are independent of $x \in \mathcal{I}_{k}$ and are entire functions. Taking into account (2.20) and (2.22), a short calculation gives

$$
\omega_{1}(\lambda)=D_{1} \omega_{2}(\lambda)=\cdots=\prod_{i=1}^{m} D_{i} \omega_{m+1}(\lambda)
$$

for each $\lambda \in \mathbb{C}$. 
Corollary 2.3 The zeros of the functions $\omega_{k}(\lambda)(k=1,2, \ldots, m+1)$ coincide.

Then we may introduce into the consideration the characteristic function $\omega(\lambda)$ as

$$
\omega(\lambda):=\omega_{1}(\lambda)=D_{1} \omega_{2}(\lambda)=\cdots=\prod_{i=1}^{m} D_{i} \omega_{m+1}(\lambda) .
$$

Lemma 2.4 All eigenvalues of problem (2.1)-(2.4) are just zeros of the function $\omega(\lambda)$.

Proof Since the functions $\varphi_{1}(x, \lambda)$ and $\varphi_{2}(x, \lambda)$ satisfy the boundary condition (2.2) and the transmission conditions (2.4), to find the eigenvalues of the (2.1)-(2.4) we have to insert the functions $\varphi_{1}(x, \lambda)$ and $\varphi_{2}(x, \lambda)$ in the boundary condition (2.3) and find the roots of this equation.

In the following lemma, we show that all eigenvalues of the problem (2.1)-(2.4) are simple.

Lemma 2.5 The eigenvalues of the boundary value problem (2.1)-(2.4) form an at most countable set without finite limit points. All eigenvalues of the boundary value problem (2.1)-(2.4) (of $\omega(\lambda))$ are simple.

Proof The eigenvalues are the zeros of the entire function occurring on the left-hand side, see equation (2.24), in

$$
\prod_{i=1}^{m} D_{i}\left[\sin \beta \varphi_{1, m+1}(b, \lambda)+\cos \beta \varphi_{2, m+1}(b, \lambda)\right]=0 .
$$

We have shown (see Lemma 2.1) that this function does not vanish for non-real $\lambda$. In particular, it does not vanish identically. Therefore, its zeros form an at most countable set without finite limit points.

By (2.1) we obtain for $\lambda, \mu \in \mathbb{C}, \lambda \neq \mu$,

$$
r(x) \frac{d}{d x}\left\{\varphi_{1}(x, \lambda) \varphi_{2}(x, \mu)-\varphi_{1}(x, \mu) \varphi_{2}(x, \lambda)\right\}=(\mu-\lambda)\left\{\varphi_{1}(x, \lambda) \varphi_{1}(x, \mu)+\varphi_{2}(x, \lambda) \varphi_{2}(x, \mu)\right\} .
$$

Integrating the above equation through $\left[a, c_{1}\right),\left(c_{k-1}, c_{k}\right)(k=2,3, \ldots, m)$ and $\left(c_{m}, b\right]$, we obtain

$$
\begin{aligned}
& \frac{\mu-\lambda}{r_{1}}\left[\int_{a}^{c_{1}}\left(\varphi_{1,1}(x, \lambda) \varphi_{1,1}(x, \mu)+\varphi_{2,1}(x, \lambda) \varphi_{2,1}(x, \mu)\right) d x\right] \\
& =\varphi_{1,1}\left(c_{1}^{-}, \lambda\right) \varphi_{2,1}\left(c_{1}^{-}, \mu\right)-\varphi_{1,1}\left(c_{1}^{-}, \lambda\right) \varphi_{2,1}\left(c_{1}^{-}, \mu\right) \\
& \quad-\left(\varphi_{1,1}(a, \lambda) \varphi_{2,1}(a, \mu)-\varphi_{1,1}(a, \lambda) \varphi_{2,1}(a, \mu)\right), \\
& \frac{\mu-\lambda}{r_{k}}\left[\int_{c_{k-1}}^{c_{k}}\left(\varphi_{1, k}(x, \lambda) \varphi_{1, k}(x, \mu)+\varphi_{2, k}(x, \lambda) \varphi_{2, k}(x, \mu)\right) d x\right] \\
& =\varphi_{1, k}\left(c_{k}^{-}, \lambda\right) \varphi_{2, k}\left(c_{k}^{-}, \mu\right)-\varphi_{1, k}\left(c_{k}^{-}, \lambda\right) \varphi_{2, k}\left(c_{k}^{-}, \mu\right) \\
& \quad-\left[\varphi_{1, k}\left(c_{k-1}^{+}, \lambda\right) \varphi_{2, k}\left(c_{k-1}^{+}, \mu\right)-\varphi_{1, k}\left(c_{k-1}^{+}, \lambda\right) \varphi_{2, k}\left(c_{k-1}^{+}, \mu\right)\right],
\end{aligned}
$$


for $k=2,3, \ldots, m$, and

$$
\begin{aligned}
\frac{\mu-\lambda}{r_{m+1}} & {\left[\int_{c_{m}}^{b}\left(\varphi_{1, m+1}(x, \lambda) \varphi_{1, m+1}(x, \mu)+\varphi_{2, m+1}(x, \lambda) \varphi_{2, m+1}(x, \mu)\right) d x\right] } \\
= & \varphi_{1, m+1}(b, \lambda) \varphi_{2, m+1}(b, \mu)-\varphi_{1, m+1}(b, \lambda) \varphi_{2, m+1}(b, \mu) \\
& -\left[\varphi_{1, m+1}\left(c_{m}^{+}, \lambda\right) \varphi_{2, m+1}\left(c_{m}^{+}, \mu\right)-\varphi_{1, m+1}\left(c_{m}^{+}, \lambda\right) \varphi_{2, m+1}\left(c_{m}^{+}, \mu\right)\right] .
\end{aligned}
$$

Then from (2.16) and the transmission conditions (2.20), we have

$$
\begin{aligned}
& \frac{\mu-\lambda}{r_{1}}\left[\int_{a}^{c_{1}}\left(\varphi_{1,1}(x, \lambda) \varphi_{1,1}(x, \mu)+\varphi_{2,1}(x, \lambda) \varphi_{2,1}(x, \mu)\right) d x\right] \\
& \quad+(\mu-\lambda) \sum_{k=2}^{m} \frac{\prod_{i=1}^{k-1} D_{i}}{r_{k}}\left[\int_{c_{k-1}}^{c_{k}}\left(\varphi_{1, k}(x, \lambda) \varphi_{1, k}(x, \mu)+\varphi_{2, k}(x, \lambda) \varphi_{2, k}(x, \mu)\right) d x\right] \\
& \quad+(\mu-\lambda)\left[\frac{\prod_{i=1}^{m} D_{i}}{r_{m+1}} \int_{c_{m}}^{b}\left(\varphi_{1, m+1}(x, \lambda) \varphi_{1, m+1}(x, \mu)+\varphi_{2, m+1}(x, \lambda) \varphi_{2, m+1}(x, \mu)\right) d x\right] \\
& =\prod_{i=1}^{m} D_{i}\left[\varphi_{1, m+1}(b, \lambda) \varphi_{2, m+1}(b, \mu)-\varphi_{1, m+1}(b, \lambda) \varphi_{2, m+1}(b, \mu)\right] .
\end{aligned}
$$

Dividing both sides of (2.28) by $(\lambda-\mu)$ and by letting $\mu \rightarrow \lambda$, we arrive at the relation

$$
\begin{aligned}
-\frac{1}{r_{1}}\left[\int_{a}^{c_{1}}\left(\left|\varphi_{1,1}(x, \lambda)\right|^{2}+\left|\varphi_{2,1}(x, \lambda)\right|^{2}\right) d x\right] \\
\quad-\sum_{k=2}^{m} \frac{\prod_{i=1}^{k-1} D_{i}}{r_{k}}\left[\int_{c_{k-1}}^{c_{k}}\left(\left|\varphi_{1, k}(x, \lambda)\right|^{2}+\left|\varphi_{2, k}(x, \lambda)\right|^{2}\right) d x\right] \\
\quad-\frac{\prod_{i=1}^{m} D_{i}}{r_{m+1}}\left[\int_{c_{m}}^{b}\left(\left|\varphi_{1, m+1}(x, \lambda)\right|^{2}+\left|\varphi_{2, m+1}(x, \lambda)\right|^{2}\right) d x\right] \\
=\prod_{i=1}^{m} D_{i}\left(\varphi_{2, m+1}(b, \lambda) \frac{\partial \varphi_{1, m+1}(b, \lambda)}{\partial \lambda}-\varphi_{1, m+1}(b, \lambda) \frac{\partial \varphi_{2, m+1}(b, \lambda)}{\partial \lambda}\right) .
\end{aligned}
$$

We show that the equation

$$
\omega(\lambda)=-\prod_{i=1}^{m} D_{i}\left(\sin \beta \varphi_{1, m+1}(b, \lambda)+\cos \beta \varphi_{2, m+1}(b, \lambda)\right)=0
$$

has only simple roots. Assume the converse, i.e., equation (2.30) has a double root $\lambda^{*}$, say. Then the following two equations hold:

$$
\begin{aligned}
& \sin \beta \varphi_{1, m+1}\left(b, \lambda^{*}\right)+\cos \beta \varphi_{2, m+1}\left(b, \lambda^{*}\right)=0, \\
& \sin \beta \frac{\partial \varphi_{1, m+1}\left(b, \lambda^{*}\right)}{\partial \lambda}+\cos \beta \frac{\partial \varphi_{2, m+1}\left(b, \lambda^{*}\right)}{\partial \lambda}=0 .
\end{aligned}
$$

Equations (2.31) and (2.32) imply that

$$
\varphi_{2, m+1}\left(b, \lambda^{*}\right) \frac{\partial \varphi_{1, m+1}\left(b, \lambda^{*}\right)}{\partial \lambda}-\varphi_{1, m+1}\left(b, \lambda^{*}\right) \frac{\partial \varphi_{2, m+1}\left(b, \lambda^{*}\right)}{\partial \lambda}=0 .
$$


Combining (2.33) and (2.29), with $\lambda=\lambda^{*}$, we obtain

$$
\begin{aligned}
& \frac{1}{r_{1}}\left[\int_{a}^{c_{1}}\left(\left|\varphi_{1,1}(x, \lambda)\right|^{2}+\left|\varphi_{2,1}(x, \lambda)\right|^{2}\right) d x\right] \\
& \quad+\sum_{k=2}^{m} \frac{\prod_{i=1}^{k-1} D_{i}}{r_{k}}\left[\int_{c_{k-1}}^{c_{k}}\left(\left|\varphi_{1, k}(x, \lambda)\right|^{2}+\left|\varphi_{2, k}(x, \lambda)\right|^{2}\right) d x\right] \\
& \quad+\frac{\prod_{i=1}^{m} D_{i}}{r_{m+1}}\left[\int_{c_{m}}^{b}\left(\left|\varphi_{1, m+1}(x, \lambda)\right|^{2}+\left|\varphi_{2, m+1}(x, \lambda)\right|^{2}\right) d x\right]=0 .
\end{aligned}
$$

It follows that $\varphi_{1}\left(x, \lambda^{*}\right)=\varphi_{2}\left(x, \lambda^{*}\right)=0$, which is impossible. This proves the lemma.

Here $\left\{\varphi\left(\cdot, \lambda_{n}\right)\right\}_{n=-\infty}^{\infty}$ will be a sequence of vector-valued eigenfunctions of (2.1)-(2.4) corresponding to the eigenvalues $\left\{\lambda_{n}\right\}_{n=-\infty}^{\infty}$. Since $\chi(\cdot, \lambda)$ satisfies (2.3) and (2.4), then the eigenvalues are also determined via

$$
\sin \alpha \chi_{1,1}(a, \lambda)+\cos \alpha \chi_{2,1}(a, \lambda)=\omega(\lambda) .
$$

Therefore $\left\{\chi\left(\cdot, \lambda_{n}\right)\right\}_{n=-\infty}^{\infty}$ is another set of vector-valued eigenfunctions which is related by $\left\{\varphi\left(\cdot, \lambda_{n}\right)\right\}_{n=-\infty}^{\infty}$ with

$$
\chi\left(x, \lambda_{n}\right)=\tau_{n} \varphi\left(x, \lambda_{n}\right), \quad x \in\left[a, c_{1}\right) \cup\left(c_{1}, c_{2}\right) \cup\left(c_{2}, c_{3}\right) \cup \cdots \cup\left(c_{m}, b\right], n \in \mathbb{Z},
$$

where $\tau_{n} \neq 0$ are non-zero constants, since all eigenvalues are simple. Since the eigenvalues are all real, we can take the vector-valued eigenfunctions to be real valued.

\section{Asymptotic formulas of eigenvalues and eigenvector functions}

In this section, we derive the asymptotic formulas of the eigenvalues $\left\{\lambda_{n}\right\}_{n=-\infty}^{\infty}$ and the vector-valued eigenfunctions $\left\{\varphi\left(\cdot, \lambda_{n}\right)\right\}_{n=-\infty}^{\infty}$. We shall transform equations (2.1), (2.13), (2.16), and (2.20) into the integral equations, see [2],

$$
\begin{aligned}
\varphi_{1,1}(x, \lambda)= & \cos \left[\frac{\lambda(x-a)}{r_{1}}-\alpha\right]-\frac{1}{r_{1}} \int_{a}^{x} \sin \left[\frac{\lambda(x-t)}{r_{1}}\right] q_{1}(t) \varphi_{1,1}(t, \lambda) d t \\
& -\frac{1}{r_{1}} \int_{a}^{x} \cos \left[\frac{\lambda(x-t)}{r_{1}}\right] q_{2}(t) \varphi_{2,1}(t, \lambda) d t, \\
\varphi_{2,1}(x, \lambda)= & \sin \left[\frac{\lambda(x-a)}{r_{1}}-\alpha\right]+\frac{1}{r_{1}} \int_{a}^{x} \cos \left[\frac{\lambda(x-t)}{r_{1}}\right] q_{1}(t) \varphi_{1,1}(t, \lambda) d t \\
& -\frac{1}{r_{1}} \int_{a}^{x} \sin \left[\frac{\lambda(x-t)}{r_{1}}\right] q_{2}(t) \varphi_{2,1}(t, \lambda) d t, \\
\varphi_{1, k+1}(x, \lambda)= & \frac{\gamma_{k}}{\delta_{k}} \varphi_{1, k}\left(c_{k}^{-}, \lambda\right) \cos \left[\frac{\lambda\left(x-c_{k}\right)}{r_{k+1}}\right]-\frac{\gamma_{k}^{\prime}}{\delta_{k}^{\prime}} \varphi_{2, k}\left(c_{k}^{-}, \lambda\right) \sin \left[\frac{\lambda\left(x-c_{k}\right)}{r_{k+1}}\right] \\
& -\frac{1}{r_{k+1}} \int_{c_{k}}^{x} \sin \left[\frac{\lambda(x-t)}{r_{k+1}}\right] q_{1}(t) \varphi_{1, k+1}(t, \lambda) d t \\
& -\frac{1}{r_{k+1}} \int_{c_{k}}^{x} \cos \left[\frac{\lambda(x-t)}{r_{k+1}}\right] q_{2}(t) \varphi_{2, k+1}(t, \lambda) d t,
\end{aligned}
$$




$$
\begin{aligned}
\varphi_{2, k+1}(x, \lambda)= & \frac{\gamma_{k}}{\delta_{k}} \varphi_{1, k}\left(c_{k}^{-}, \lambda\right) \sin \left[\frac{\lambda\left(x-c_{k}\right)}{r_{k+1}}\right]+\frac{\gamma_{k}^{\prime}}{\delta_{k}^{\prime}} \varphi_{2, k}\left(c_{k}^{-}, \lambda\right) \cos \left[\frac{\lambda\left(x-c_{k}\right)}{r_{k+1}}\right] \\
& +\frac{1}{r_{k+1}} \int_{c_{k}}^{x} \cos \left[\frac{\lambda(x-t)}{r_{k+1}}\right] q_{1}(t) \varphi_{1, k+1}(t, \lambda) d t \\
& -\frac{1}{r_{k+1}} \int_{c_{k}}^{x} \sin \left[\frac{\lambda(x-t)}{r_{k+1}}\right] q_{2}(t) \varphi_{2, k+1}(t, \lambda) d t,
\end{aligned}
$$

where $k=1,2, \ldots, m$.

For $|\lambda| \rightarrow \infty$ the following estimates hold uniformly with respect to $x, x \in\left[a, c_{1}\right) \cup$ $\left(c_{1}, c_{2}\right) \cup\left(c_{2}, c_{3}\right) \cup \cdots \cup\left(c_{m}, b\right], c f .[1]$, p.55:

$$
\begin{aligned}
& \varphi_{1,1}(x, \lambda)=\cos \left[\frac{\lambda(x-a)}{r_{1}}-\alpha\right]+\mathcal{O}\left(\frac{1}{\lambda}\right), \\
& \varphi_{2,1}(x, \lambda)=\sin \left[\frac{\lambda(x-a)}{r_{1}}-\alpha\right]+\mathcal{O}\left(\frac{1}{\lambda}\right), \\
& \varphi_{1, k+1}(x, \lambda)=\frac{\gamma_{k}}{\delta_{k}} \varphi_{1, k}\left(c_{k}^{-}, \lambda\right) \cos \left[\frac{\lambda\left(x-c_{k}\right)}{r_{k+1}}\right]-\frac{\gamma_{k}^{\prime}}{\delta_{k}^{\prime}} \varphi_{2, k}\left(c_{k}^{-}, \lambda\right) \sin \left[\frac{\lambda\left(x-c_{k}\right)}{r_{k+1}}\right] \\
&+ \mathcal{O}\left(\frac{1}{\lambda}\right), \quad k=1,2, \ldots, m, \\
& \varphi_{2, k+1}(x, \lambda)= \frac{\gamma_{k}}{\delta_{k}} \varphi_{1, k}\left(c_{k}^{-}, \lambda\right) \sin \left[\frac{\lambda\left(x-c_{k}\right)}{r_{k+1}}\right]+\frac{\gamma_{k}^{\prime}}{\delta_{k}^{\prime}} \varphi_{2, k}\left(c_{k}^{-}, \lambda\right) \cos \left[\frac{\lambda\left(x-c_{k}\right)}{r_{k+1}}\right] \\
&+\mathcal{O}\left(\frac{1}{\lambda}\right), \quad k=1,2, \ldots, m .
\end{aligned}
$$

Now we will find an asymptotic formula for the eigenvalues. Let $\gamma_{k} \delta_{k}^{\prime}-\gamma_{k}^{\prime} \delta_{k}=0, k=$ $1,2, \ldots, m$. Since the eigenvalues of the boundary value problem (2.1)-(2.4) coincide with the roots of the equation

$$
\prod_{k=1}^{m} D_{k}\left[\sin \beta \varphi_{1, m+1}(b, \lambda)+\cos \beta \varphi_{2, m+1}(b, \lambda)\right]=0,
$$

then from the estimates (3.7) and (3.8) (with $k=m$ ) in (3.9) we get

$$
\begin{aligned}
& \sin \beta\left[\frac{\gamma_{m}}{\delta_{m}} \varphi_{1, m}\left(c_{m}^{-}, \lambda\right) \cos \left[\frac{\lambda\left(b-c_{m}\right)}{r_{m+1}}\right]-\frac{\gamma_{m}^{\prime}}{\delta_{m}^{\prime}} \varphi_{2, m}\left(c_{m}^{-}, \lambda\right) \sin \left[\frac{\lambda\left(b-c_{m}\right)}{r_{m+1}}\right]\right] \\
& +\cos \beta\left[\frac{\gamma_{m}}{\delta_{m}} \varphi_{1, m}\left(c_{m}^{-}, \lambda\right) \sin \left[\frac{\lambda\left(b-c_{m}\right)}{r_{m+1}}\right]+\frac{\gamma_{m}^{\prime}}{\delta_{m}^{\prime}} \varphi_{2, m}\left(c_{m}^{-}, \lambda\right) \cos \left[\frac{\lambda\left(b-c_{m}\right)}{r_{m+1}}\right]\right] \\
& +\mathcal{O}\left(\frac{1}{\lambda}\right)=0
\end{aligned}
$$

which can be written as

$$
\begin{aligned}
& \frac{\gamma_{m}}{\delta_{m}} \varphi_{1, m}\left(c_{m}^{-}, \lambda\right) \sin \left[\frac{\lambda\left(b-c_{m}\right)}{r_{m+1}}+\beta\right]+\frac{\gamma_{m}^{\prime}}{\delta_{m}^{\prime}} \varphi_{2, m}\left(c_{m}^{-}, \lambda\right) \cos \left[\frac{\lambda\left(b-c_{m}\right)}{r_{m+1}}+\beta\right] \\
& \quad+\mathcal{O}\left(\frac{1}{\lambda}\right)=0 .
\end{aligned}
$$


Then, from (3.5), (3.6), (3.7), (3.8), and $\gamma_{k} \delta_{k}^{\prime}-\gamma_{k}^{\prime} \delta_{k}=0$, equation (3.10) has the form

$$
\begin{aligned}
& \sin \left[\frac{\lambda\left(c_{1}-a\right)}{r_{1}}+\frac{\lambda\left(c_{2}-c_{1}\right)}{r_{2}}+\frac{\lambda\left(c_{3}-c_{2}\right)}{r_{3}}+\cdots+\frac{\lambda\left(c_{m}-c_{m-1}\right)}{r_{m}}+\frac{\lambda\left(b-c_{m}\right)}{r_{m+1}}-\alpha+\beta\right] \\
& \quad+\mathcal{O}\left(\frac{1}{\lambda}\right)=0,
\end{aligned}
$$

which can be rewritten as

$$
\sin [\Theta \lambda-\alpha+\beta]+\mathcal{O}\left(\frac{1}{\lambda}\right)=0, \quad \Theta:=\sum_{k=0}^{m} \frac{\left(c_{k+1}-c_{k}\right)}{r_{k+1}}
$$

For large $|\lambda|$ equation (3.12) obviously has solutions which, as is not hard to see, have the form, $c f .[1]$, p.57,

$$
\Theta \lambda_{n}-\alpha+\beta=n \pi+\delta_{n}, \quad n=0, \pm 1, \pm 2, \ldots
$$

Inserting these values in (3.12), we find that $\sin \delta_{n}=\mathcal{O}\left(\frac{1}{n}\right)$, i.e., $\delta_{n}=\mathcal{O}\left(\frac{1}{n}\right)$. Thus we obtain the following asymptotic formula for the eigenvalues:

$$
\lambda_{n}=\frac{n \pi+\alpha-\beta}{\Theta}+\mathcal{O}\left(\frac{1}{n}\right), \quad n=0, \pm 1, \pm 2, \ldots
$$

Using equations (3.14), we obtain the following asymptotic formulas for the vector-valued eigenfunctions $\varphi\left(\cdot, \lambda_{n}\right)$, where $\gamma_{k} \delta_{k}^{\prime}-\gamma_{k}^{\prime} \delta_{k}=0$ :

$$
\varphi\left(x, \lambda_{n}\right)= \begin{cases}\left(\begin{array}{ll}
\varphi_{1,1}\left(x, \lambda_{n}\right)=\cos \left[\frac{\lambda_{n}(x-a)}{r_{1}}-\alpha\right]+\mathcal{O}\left(\frac{1}{n}\right) \\
\varphi_{2,1}\left(x, \lambda_{n}\right)=\sin \left[\frac{\lambda_{n}(x-a)}{r_{1}}-\alpha\right]+\mathcal{O}\left(\frac{1}{n}\right)
\end{array}\right), & x \in\left[c_{0}, c_{1}\right), \\
\left(\begin{array}{ll}
\varphi_{1, k+1}\left(x, \lambda_{n}\right)=\prod_{i=1}^{k} \frac{\gamma_{k}}{\delta_{k}} \cos \left[\lambda_{n} \sum_{i=1}^{k} \frac{\left(c_{i}-c_{i-1}\right)}{r_{i}}+\frac{\lambda_{n}\left(x-c_{k}\right)}{r_{k+1}}-\alpha\right]+\mathcal{O}\left(\frac{1}{n}\right) \\
\varphi_{2, k+1}\left(x, \lambda_{n}\right)=\prod_{i=1}^{k} \frac{\gamma_{k}}{\delta_{k}} \sin \left[\lambda_{n} \sum_{i=1}^{k} \frac{\left(c_{i}-c_{i-1}\right)}{r_{i}}+\frac{\lambda_{n}\left(x-c_{k}\right)}{r_{k+1}}-\alpha\right]+\mathcal{O}\left(\frac{1}{n}\right)
\end{array}\right), & x \in\left(c_{k}, c_{k+1}\right),\end{cases}
$$

where $k=1,2, \ldots, m$.

\section{Green's matrix and expansion theorem}

Let $f(\cdot)=\left(\begin{array}{l}f_{1}(\cdot) \\ f_{2}(\cdot)\end{array}\right)$ be a continuous vector-valued function. Now, we derive the Green's matrix of problem (2.1)-(2.4). Consider the inhomogeneous eigenvalue problem consisting of the differential system

$$
\begin{aligned}
& r(x) u_{2}^{\prime}(x)-\left\{q_{1}(x)+\lambda\right\} u_{1}(x)=f_{1}(x), \\
& \left.r(x) u_{1}^{\prime}(x)+\left\{q_{2}(x)+\lambda\right\} u_{2}(x)=-f_{2}(x),\right\} \\
& x \in\left[a, c_{1}\right) \cup\left(c_{1}, c_{2}\right) \cup\left(c_{2}, c_{3}\right) \cup \cdots \cup\left(c_{m}, b\right],
\end{aligned}
$$

and the boundary conditions (2.2)-(2.4) with $\lambda$ is not an eigenvalue of problem (2.1)-(2.4).

Now, we can represent the general solution of (4.1) in the following form:

$$
u(x, \lambda)= \begin{cases}\mathcal{A}_{1}(x, \lambda)\left(\begin{array}{l}
\varphi_{1,1}(x, \lambda) \\
\varphi_{2,1}(x, \lambda)
\end{array}\right)+\mathcal{B}_{1}(x, \lambda)\left(\begin{array}{l}
\chi_{1,1}(x, \lambda) \\
\chi_{2,1}(x, \lambda)
\end{array}\right), & x \in\left[a, c_{1}\right), \\
\mathcal{A}_{k}(x, \lambda)\left(\begin{array}{l}
\varphi_{1, k}(x, \lambda) \\
\varphi_{2, k}(x, \lambda)
\end{array}\right)+\mathcal{B}_{k}(x, \lambda)\left(\begin{array}{l}
\chi_{1, k}(x, \lambda) \\
\chi_{2, k}(x, \lambda)
\end{array}\right), & x \in\left(c_{k-1}, c_{k}\right)(k=2,3, \ldots, m), \\
\mathcal{A}_{m+1}(x, \lambda)\left(\begin{array}{c}
\varphi_{1, m+1}(x, \lambda) \\
\varphi_{2, m+1}(x, \lambda)
\end{array}\right) & x \in\left(c_{m}, b\right] . \\
\quad+\mathcal{B}_{m+1}(x, \lambda)\left(\begin{array}{l}
\chi_{1, m+1}(x, \lambda) \\
\chi_{2, m+1}(x, \lambda)
\end{array}\right), & \end{cases}
$$


We applied the standard method of variation of the constants to (4.2), thus, the functions $\mathcal{A}_{k}(x, \lambda)$ and $\mathcal{B}_{k}(x, \lambda)(k=1,2, \ldots, m+1)$ satisfy the linear system of equations

$$
\begin{aligned}
& \left.\begin{array}{l}
\mathcal{A}_{1}^{\prime}(x, \lambda) \varphi_{2,1}(x, \lambda)+\mathcal{B}_{1}^{\prime}(x, \lambda) \chi_{2,1}(x, \lambda)=\frac{f_{1}(x)}{r_{1}}, \\
\mathcal{A}_{1}^{\prime}(x, \lambda) \varphi_{1,1}(x, \lambda)+\mathcal{B}_{1}^{\prime}(x, \lambda) \chi_{1,1}(x, \lambda)=-\frac{f_{2}(x)}{r_{1}},
\end{array}\right\} \quad x \in\left[a, c_{1}\right), \\
& \left.\begin{array}{l}
\mathcal{A}_{k}^{\prime}(x, \lambda) \varphi_{2, k}(x, \lambda)+\mathcal{B}_{k}^{\prime}(x, \lambda) \chi_{2, k}(x, \lambda)=\frac{f_{1}(x)}{r_{k}}, \\
\mathcal{A}_{k}^{\prime}(x, \lambda) \varphi_{1, k}(x, \lambda)+\mathcal{B}_{k}^{\prime}(x, \lambda) \chi_{1, k}(x, \lambda)=-\frac{f_{2}(x)}{r_{k}},
\end{array}\right\} \quad x \in\left(c_{k-1}, c_{k}\right)(k=2,3, \ldots, m),
\end{aligned}
$$

and

$$
\left.\begin{array}{l}
\mathcal{A}_{m+1}^{\prime}(x, \lambda) \varphi_{2, m+1}(x, \lambda)+\mathcal{B}_{m+1}^{\prime}(x, \lambda) \chi_{2, m+1}(x, \lambda)=\frac{f_{1}(x)}{r_{m+1}}, \\
\mathcal{A}_{m+1}^{\prime}(x, \lambda) \varphi_{1, m+1}(x, \lambda)+\mathcal{B}_{m+1}^{\prime}(x, \lambda) \chi_{1, m+1}(x, \lambda)=-\frac{f_{2}(x)}{r_{m+1}},
\end{array}\right\} \quad x \in\left(c_{m}, b\right] .
$$

Since $\lambda$ is not an eigenvalue and $\omega(\lambda) \neq 0$, each of the linear system in (4.3), (4.4), and (4.5) has a unique solution which leads to

$$
\begin{aligned}
& \left.\begin{array}{l}
\mathcal{A}_{1}(x, \lambda)=\frac{1}{r_{1} \omega_{1}(\lambda)} \int_{x}^{c_{1}} \chi^{\top}(\xi, \lambda) f(\xi) d \xi+\mathcal{A}_{1}, \\
\mathcal{B}_{1}(x, \lambda)=\frac{1}{r_{1} \omega_{1}(\lambda)} \int_{a}^{x} \varphi^{\top}(\xi, \lambda) f(\xi) d \xi+\mathcal{B}_{1},
\end{array}\right\} \quad x \in\left[a, c_{1}\right), \\
& \left.\begin{array}{l}
\mathcal{A}_{k}(x, \lambda)=\frac{1}{r_{k} \omega_{k}(\lambda)} \int_{x}^{c_{k}} \chi^{\top}(\xi, \lambda) f(\xi) d \xi+\mathcal{A}_{k}, \\
\mathcal{B}_{k}(x, \lambda)=\frac{1}{r_{k} \omega_{k}(\lambda)} \int_{c_{k-1}}^{x} \varphi^{\top}(\xi, \lambda) f(\xi) d \xi+\mathcal{B}_{k},
\end{array}\right\} \quad x \in\left(c_{k-1}, c_{k}\right)(k=2,3, \ldots, m), \\
& \left.\begin{array}{l}
\mathcal{A}_{m+1}(x, \lambda)=\frac{1}{r_{m+1} \omega_{m+1}(\lambda)} \int_{x}^{b} \chi^{\top}(\xi, \lambda) f(\xi) d \xi+\mathcal{A}_{m+1}, \\
\mathcal{B}_{m+1}(x, \lambda)=\frac{1}{r_{m+1} \omega_{m+1}(\lambda)} \int_{c_{m}}^{x} \varphi^{\top}(\xi, \lambda) f(\xi) d \xi+\mathcal{B}_{m+1},
\end{array}\right\} \quad x \in\left(c_{m}, b\right],
\end{aligned}
$$

where $\mathcal{A}_{k}$ and $\mathcal{B}_{k}, k=1,2, \ldots, m+1$, are arbitrary constants, and

$$
\begin{aligned}
\varphi(\xi, \lambda)= \begin{cases}\left(\begin{array}{l}
\varphi_{1,1}(\xi, \lambda) \\
\varphi_{2,1}(\xi, \lambda)
\end{array}\right), & \xi \in\left[a, c_{1}\right), \\
\left(\begin{array}{ll}
\varphi_{1, k}(\xi, \lambda) \\
\varphi_{2, k}(\xi, \lambda)
\end{array}\right), & \xi \in\left(c_{k-1}, c_{k}\right)(k=2,3, \ldots, m), \\
\left(\begin{array}{ll}
\varphi_{1, m+1}(\xi, \lambda) \\
\varphi_{2, m+1}(\xi, \lambda)
\end{array}\right), & \xi \in\left(c_{m}, b\right],\end{cases} \\
\chi(\xi, \lambda)= \begin{cases}\left(\begin{array}{l}
\chi_{1,1}(\xi, \lambda) \\
\chi_{2,1}(\xi, \lambda)
\end{array}\right), & \xi \in\left[a, c_{1}\right), \\
\left(\begin{array}{ll}
\chi_{1, k}(\xi, \lambda) \\
\chi_{2, k}(\xi, \lambda)
\end{array}\right), & \xi \in\left(c_{k-1}, c_{k}\right)(k=2,3, \ldots, m), \\
\left(\begin{array}{c}
\chi_{1, m+1}(\xi, \lambda) \\
\chi_{2, m+1}(\xi, \lambda)
\end{array}\right), & \xi \in\left(c_{m}, b\right] .\end{cases}
\end{aligned}
$$

Substituting equations (4.6), (4.7), and (4.8) into (4.2), we obtain the solution of (4.1),

$$
u(x, \lambda)=\left\{\begin{array}{l}
\frac{\varphi(x, \lambda)}{r_{1} \omega_{1}(\lambda)} \int_{x}^{c_{1}} \chi^{\top}(\xi, \lambda) f(\xi) d \xi+\frac{\chi(x, \lambda)}{r_{1} \omega_{1}(\lambda)} \int_{a}^{x} \varphi^{\top}(\xi, \lambda) f(\xi) d \xi \\
\quad+\mathcal{A}_{1} \varphi(x, \lambda)+\mathcal{B}_{1} \chi(x, \lambda), \quad x \in\left[a, c_{1}\right), \\
\frac{\varphi(x, \lambda)}{r_{k} \omega_{k}(\lambda)} \int_{x}^{c_{k}} \chi^{\top}(\xi, \lambda) f(\xi) d \xi+\frac{\chi(x, \lambda)}{r_{k} \omega_{k}(\lambda)} \int_{c_{k-1}}^{x} \varphi^{\top}(\xi, \lambda) f(\xi) d \xi \\
\quad+\mathcal{A}_{k} \varphi(x, \lambda)+\mathcal{B}_{k} \chi(x, \lambda), \quad x \in\left(c_{k-1}, c_{k}\right)(k=2,3, \ldots, m), \\
\frac{\varphi(x, \lambda)}{r_{m+1} \omega_{m+1}(\lambda)} \int_{x}^{b} \chi^{\top}(\xi, \lambda) f(\xi) d \xi+\frac{\chi(x, \lambda)}{r_{m+1} \omega_{m+1}(\lambda)} \int_{c_{m}}^{x} \varphi^{\top}(\xi, \lambda) f(\xi) d \xi \\
\quad+\mathcal{A}_{m+1} \varphi(x, \lambda)+\mathcal{B}_{m+1} \chi(x, \lambda), \quad x \in\left(c_{m}, b\right] .
\end{array}\right.
$$


Then from (2.2), (2.3), and (2.4), we get

$$
\begin{aligned}
& \left\{\begin{aligned}
& \mathcal{A}_{1}= \frac{1}{r_{2} \omega_{2}(\lambda)} \int_{c_{1}}^{c_{2}} \chi^{\top}(\xi, \lambda) f(\xi) d \xi+\frac{1}{r_{3} \omega_{3}(\lambda)} \int_{c_{2}}^{c_{3}} \chi^{\top}(\xi, \lambda) f(\xi) d \xi \\
&+\cdots+\frac{1}{r_{m+1} \omega_{m+1}(\lambda)} \int_{c_{m}}^{b} \chi^{\top}(\xi, \lambda) f(\xi) d \xi, \\
& \mathcal{A}_{2}= \frac{1}{r_{3} \omega_{3}(\lambda)} \int_{c_{2}}^{c_{3}} \chi^{\top}(\xi, \lambda) f(\xi) d \xi+\frac{1}{r_{4} \omega_{4}(\lambda)} \int_{c_{3}}^{c_{4}} \chi^{\top}(\xi, \lambda) f(\xi) d \xi \\
&+\cdots+\frac{1}{r_{m+1} \omega_{m+1}(\lambda)} \int_{c_{m}}^{b} \chi^{\top}(\xi, \lambda) f(\xi) d \xi, \\
& \ldots, \\
& \mathcal{A}_{m}= \frac{1}{r_{m+1} \omega_{m+1}(\lambda)} \int_{c_{m}}^{b} \chi^{\top}(\xi, \lambda) f(\xi) d \xi, \\
& \mathcal{A}_{m+1}=0,
\end{aligned}\right. \\
& \begin{cases}\mathcal{B}_{1}= & 0, \\
\mathcal{B}_{2}= & \frac{1}{r_{1} \omega_{1}(\lambda)} \int_{a}^{c_{1}} \varphi^{\top}(\xi, \lambda) f(\xi) d \xi, \\
\mathcal{B}_{3}= & \frac{1}{r_{1} \omega_{1}(\lambda)} \int_{a}^{c_{1}} \varphi^{\top}(\xi, \lambda) f(\xi) d \xi+\frac{1}{r_{2} \omega_{2}(\lambda)} \int_{c_{1}}^{c_{2}} \varphi^{\top}(\xi, \lambda) f(\xi) d \xi, \\
\ldots, & \quad+\cdots+\frac{1}{r_{m} \omega_{m}(\lambda)} \int_{c_{m-1}}^{c_{m}} \varphi^{\top}(\xi, \lambda) f(\xi) d \xi . \\
\mathcal{B}_{m+1}= & \frac{1}{r_{1} \omega_{1}(\lambda)} \int_{a}^{c_{1}} \varphi^{\top}(\xi, \lambda) f(\xi) d \xi+\frac{1}{r_{2} \omega_{2}(\lambda)} \int_{c_{1}}^{c_{2}} \varphi^{\top}(\xi, \lambda) f(\xi) d \xi\end{cases}
\end{aligned}
$$

Equations (4.10) and (4.11) can be rewritten as follows:

$$
\mathcal{A}_{k}:= \begin{cases}\sum_{i=k}^{m} \frac{1}{r_{i+1} \omega_{i+1}(\lambda)} \int_{c_{i}}^{c_{i+1}} \chi^{\top}(\xi, \lambda) f(\xi) d \xi, & k=1,2, \ldots, m \\ 0, & k=m+1\end{cases}
$$

and

$$
\mathcal{B}_{k}:= \begin{cases}0, & k=1, \\ \sum_{i=1}^{k-1} \frac{1}{r_{i} \omega_{i}(\lambda)} \int_{c_{i-1}}^{c_{i}} \varphi^{\top}(\xi, \lambda) f(\xi) d \xi, & k=2,3, \ldots, m+1,\end{cases}
$$

respectively. Then, from (4.12) and (4.13), (4.9) can be written as

$$
u(x, \lambda)=\left\{\begin{array}{l}
\frac{\varphi(x, \lambda)}{r_{1} \omega_{1}(\lambda)} \int_{x}^{c_{1}} \chi^{\top}(\xi, \lambda) f(\xi) d \xi+\frac{\chi(x, \lambda)}{r_{1} \omega_{1}(\lambda)} \int_{a}^{x} \varphi^{\top}(\xi, \lambda) f(\xi) d \xi \\
\quad+\sum_{i=1}^{m} \frac{\varphi(x, \lambda)}{r_{i+1} \omega_{i+1}(\lambda)} \int_{c_{i}}^{c_{i+1}} \chi^{\top}(\xi, \lambda) f(\xi) d \xi, \quad x \in\left[a, c_{1}\right), \\
\frac{\varphi(x, \lambda)}{r_{k} \omega_{k}(\lambda)} \int_{x}^{c_{k}} \chi^{\top}(\xi, \lambda) f(\xi) d \xi+\frac{\chi(x, \lambda)}{r_{k} \omega_{k}(\lambda)} \int_{c_{k-1}}^{x} \varphi^{\top}(\xi, \lambda) f(\xi) d \xi \\
\quad+\sum_{i=k}^{m} \frac{\varphi(x, \lambda)}{r_{i+1} \omega_{i+1}(\lambda)} \int_{c_{i}}^{c_{i+1}} \chi^{\top}(\xi, \lambda) f(\xi) d \xi \\
\quad+\sum_{i=1}^{k-1} \frac{\chi(x, \lambda)}{r_{i} \omega_{i}(\lambda)} \int_{c_{i-1}}^{c_{i}} \varphi^{\top}(\xi, \lambda) f(\xi) d \xi, \quad x \in\left(c_{k-1}, c_{k}\right)(k=2,3, \ldots, m), \\
\frac{\varphi(x, \lambda)}{r_{m+1} \omega_{m+1}(\lambda)} \int_{x}^{b} \chi^{\top}(\xi, \lambda) f(\xi) d \xi+\frac{\chi(x, \lambda)}{r_{m+1} \omega_{m+1}(\lambda)} \int_{c_{m}}^{x} \varphi^{\top}(\xi, \lambda) f(\xi) d \xi \\
\quad+\sum_{i=1}^{m} \frac{\chi(x, \lambda)}{r_{i} \omega_{i}(\lambda)} \int_{c_{i-1}}^{c_{i}} \varphi^{\top}(\xi, \lambda) f(\xi) d \xi, \quad x \in\left(c_{m}, b\right],
\end{array}\right.
$$

which can be written as

$$
\begin{aligned}
u(x, \lambda)= & \frac{1}{r_{1}} \int_{a}^{c_{1}} G(x, \xi, \lambda) f(\xi) d \xi+\sum_{k=2}^{m} \frac{\prod_{i=1}^{k-1} D_{i}}{r_{k}} \int_{c_{k-1}}^{c_{k}} G(x, \xi, \lambda) f(\xi) d \xi \\
& +\frac{\prod_{i=1}^{m} D_{i}}{r_{m+1}} \int_{c_{m}}^{b} G(x, \xi, \lambda) f(\xi) d \xi
\end{aligned}
$$

where

$$
G(x, \xi, \lambda)=\frac{1}{\omega(\lambda)} \begin{cases}\chi(x, \lambda) \varphi^{\top}(\xi, \lambda), & a \leq \xi \leq x \leq b, x, \xi \neq c_{k}, k=1,2, \ldots, m \\ \varphi(x, \lambda) \chi^{\top}(\xi, \lambda), & a \leq x \leq \xi \leq b, x, \xi \neq c_{k}, k=1,2, \ldots, m .\end{cases}
$$


Expanding (4.16) we obtain the concrete form

$$
\begin{aligned}
& G(x, \xi, \lambda) \\
& \quad=\frac{1}{\omega(\lambda)} \begin{cases}\left(\begin{array}{l}
\varphi_{1}(\xi, \lambda) \chi_{1}(x, \lambda) \varphi_{2}(\xi, \lambda) \chi_{1}(x, \lambda) \\
\varphi_{1}(\xi, \lambda) \chi_{2}(x, \lambda) \varphi_{2}(\xi, \lambda) \chi_{2}(x, \lambda)
\end{array}\right), & a \leq \xi \leq x \leq b, x, \xi \neq c_{k}, k=1,2, \ldots, m, \\
\left(\begin{array}{l}
\varphi_{1}(x, \lambda) \chi_{1}(\xi, \lambda) \varphi_{1}(x, \lambda) \chi_{2}(\xi, \lambda) \\
\varphi_{2}(x, \lambda) \chi_{1}(\xi, \lambda) \varphi_{2}(x, \lambda) \chi_{2}(\xi, \lambda)
\end{array}\right), & a \leq x \leq \xi \leq b, x, \xi \neq c_{k}, k=1,2, \ldots, m .\end{cases}
\end{aligned}
$$

The matrix $G(x, \xi, \lambda)$ is called the Green's matrix of problem (2.1)-(2.4). Obviously $G(x, \xi, \lambda)$ is a meromorphic function of $\lambda$, for every $(x, \xi) \in\left(\left[a, c_{1}\right) \cup\left(c_{1}, c_{2}\right) \cup\left(c_{2}, c_{3}\right) \cup\right.$ $\left.\cdots \cup\left(c_{m}, b\right]\right)^{2}$, which has simple poles only at the eigenvalues. Although the Green's function looks as simple as that of Dirac systems, $c f$. e.g. [1, 2], it is rather complicated because of the transmission conditions; see the example at the end of this paper.

The next theorem is a vector-valued eigenfunction expansion theorem. The proof is exactly similar to that of Levitan and Sargsjan derived in [1], pp.67-77; see also [2].

\section{Theorem 4.1}

(i) $\operatorname{For} u(\cdot) \in \mathcal{H}$,

$$
\|u(\cdot)\|_{\mathcal{H}}^{2}=\sum_{n=-\infty}^{\infty}\left|\left\langle u(\cdot), \psi_{n}(\cdot)\right\rangle_{\mathcal{H}}\right|^{2}
$$

(ii) For $u(\cdot) \in \mathcal{H}$,

$$
u(x)=\sum_{n=-\infty}^{\infty}\left\langle u(\cdot), \psi_{n}(\cdot)\right\rangle_{\mathcal{H}} \psi_{n}(x)
$$

where $\psi_{n}(\cdot)=\frac{\varphi\left(\cdot, \lambda_{n}\right)}{\left\|\varphi\left(\cdot, \lambda_{n}\right)\right\|_{\mathcal{H}}}$ is the normalized vector-valued eigenfunction of the problem (2.1)-(2.4). The series is absolutely and uniformly convergent on

$\left[a, c_{1}\right) \cup\left(c_{1}, c_{2}\right) \cup\left(c_{2}, c_{3}\right) \cup \cdots \cup\left(c_{m}, b\right]$.

\section{The sampling theorems}

In this section we introduce two sampling theorems associated with problem (2.1)-(2.4) above. In the first one we define an integral transform whose kernel is $\varphi(\cdot, \lambda)$, while in the second one the sampled transform will be defined in terms of the Green's matrix. The first sampling theorem of this section associated with the boundary value problem (2.1)-(2.4) is the following theorem.

Theorem 5.1 Let $f(x)=\left(\begin{array}{l}f_{1}(x) \\ f_{2}(x)\end{array}\right) \in \mathcal{H}$. For $\lambda \in \mathbb{C}$ let

$$
\begin{aligned}
\mathcal{F}(\lambda)= & \frac{1}{r_{1}} \int_{a}^{c_{1}} f^{\top}(x) \varphi(x, \lambda) d x+\sum_{k=2}^{m} \frac{\prod_{i=1}^{k-1} D_{i}}{r_{k}} \int_{c_{k-1}}^{c_{k}} f^{\top}(x) \varphi(x, \lambda) d x \\
& +\frac{\prod_{i=1}^{m} D_{i}}{r_{m+1}} \int_{c_{m}}^{b} f^{\top}(x) \varphi(x, \lambda) d x,
\end{aligned}
$$

where $\varphi(\cdot, \lambda)$ is the solution defined in (2.13). Then $\mathcal{F}(\lambda)$ is an entire function of exponential type that can be reconstructed from its values at the points $\left\{\lambda_{n}\right\}_{n=-\infty}^{\infty}$ via the sampling 
formula

$$
\mathcal{F}(\lambda)=\sum_{n=-\infty}^{\infty} \mathcal{F}\left(\lambda_{n}\right) \frac{\omega(\lambda)}{\left(\lambda-\lambda_{n}\right) \omega^{\prime}\left(\lambda_{n}\right)}
$$

The series (5.2) converges absolutely on $\mathbb{C}$ and uniformly on any compact subset of $\mathbb{C}$. Here $\omega(\lambda)$ is the entire function defined in (2.24).

Proof Equation (5.1) can be rewritten in the form

$$
\begin{aligned}
\mathcal{F}(\lambda)= & \langle f(\cdot), \varphi(\cdot, \lambda)\rangle_{\mathcal{H}} \\
= & \frac{1}{r_{1}} \int_{a}^{c_{1}} f^{\top}(x) \varphi(x, \lambda) d x+\sum_{k=2}^{m} \frac{\prod_{i=1}^{k-1} D_{i}}{r_{k}} \int_{c_{k-1}}^{c_{k}} f^{\top}(x) \varphi(x, \lambda) d x \\
& +\frac{\prod_{i=1}^{m} D_{i}}{r_{m+1}} \int_{c_{m}}^{b} f^{\top}(x) \varphi(x, \lambda) d x, \quad \lambda \in \mathbb{C} .
\end{aligned}
$$

Since both $f(\cdot)$ and $\varphi(\cdot, \lambda)$ are in $\mathcal{H}$, they have the Fourier expansions

$$
f(x)=\sum_{n=-\infty}^{\infty} \hat{f}(n) \frac{\varphi\left(x, \lambda_{n}\right)}{\left\|\varphi\left(\cdot, \lambda_{n}\right)\right\|_{\mathcal{H}}^{2}}, \quad \varphi(x, \lambda)=\sum_{n=-\infty}^{\infty}\left\langle\varphi(\cdot, \lambda),\left.\varphi\left(\cdot, \lambda_{n}\right)\right|_{\mathcal{H}} \frac{\varphi\left(x, \lambda_{n}\right)}{\left\|\varphi\left(\cdot, \lambda_{n}\right)\right\|_{\mathcal{H}}^{2}}\right.
$$

where $\lambda \in \mathbb{C}$ and $\hat{f}(n)$ are the Fourier coefficients

$$
\begin{aligned}
\hat{f}(n)= & \left\langle f(\cdot), \varphi\left(\cdot, \lambda_{n}\right)\right\rangle_{\mathcal{H}} \\
= & \frac{1}{r_{1}} \int_{a}^{c_{1}} f^{\top}(x) \varphi\left(x, \lambda_{n}\right) d x+\sum_{k=2}^{m} \frac{\prod_{i=1}^{k-1} D_{i}}{r_{k}} \int_{c_{k-1}}^{c_{k}} f^{\top}(x) \varphi\left(x, \lambda_{n}\right) d x \\
& +\frac{\prod_{i=1}^{m} D_{i}}{r_{m+1}} \int_{c_{m}}^{b} f^{\top}(x) \varphi\left(x, \lambda_{n}\right) d x .
\end{aligned}
$$

Applying Parseval's identity to (5.3), we obtain

$$
\mathcal{F}(\lambda)=\sum_{n=-\infty}^{\infty} \mathcal{F}\left(\lambda_{n}\right) \frac{\left\langle\varphi(\cdot, \lambda), \varphi\left(\cdot, \lambda_{n}\right)\right\rangle_{\mathcal{H}}}{\left\|\varphi\left(\cdot, \lambda_{n}\right)\right\|_{\mathcal{H}}^{2}}, \quad \lambda \in \mathbb{C}
$$

Now we calculate $\left\langle\varphi(\cdot, \lambda), \varphi\left(\cdot, \lambda_{n}\right)\right\rangle_{\mathcal{H}}$ and $\left\|\varphi\left(\cdot, \lambda_{n}\right)\right\|_{\mathcal{H}}$ of $\lambda \in \mathbb{C}, n \in \mathbb{Z}$. To prove expansion (5.2) we need to show that

$$
\frac{\left\langle\varphi(\cdot, \lambda), \varphi\left(\cdot, \lambda_{n}\right)\right\rangle_{\mathcal{H}}}{\left\|\varphi\left(\cdot, \lambda_{n}\right)\right\|_{\mathcal{H}}^{2}}=\frac{\omega(\lambda)}{\left(\lambda-\lambda_{n}\right) \omega^{\prime}(\lambda)}, \quad n \in \mathbb{Z}, \lambda \in \mathbb{C}
$$

Indeed, let $\lambda \in \mathbb{C}$ and $n \in \mathbb{Z}$ be fixed. By the definition of the inner product of $\mathcal{H}$, we have

$$
\begin{aligned}
\left\langle\varphi(\cdot, \lambda), \varphi\left(\cdot, \lambda_{n}\right)\right\rangle_{\mathcal{H}}= & \frac{1}{r_{1}} \int_{a}^{c_{1}} \varphi^{\top}(x, \lambda) \varphi\left(x, \lambda_{n}\right) d x+\sum_{k=2}^{m} \frac{\prod_{i=1}^{k-1} D_{i}}{r_{k}} \int_{c_{k-1}}^{c_{k}} \varphi^{\top}(x, \lambda) \varphi\left(x, \lambda_{n}\right) d x \\
& +\frac{\prod_{i=1}^{m} D_{i}}{r_{m+1}} \int_{c_{m}}^{b} \varphi^{\top}(x, \lambda) \varphi\left(x, \lambda_{n}\right) d x
\end{aligned}
$$


From the Green's identity, see [1], p.51, we have

$$
\begin{aligned}
\left(\lambda_{n}-\right. & \lambda)\left(\frac{1}{r_{1}} \int_{a}^{c_{1}} \varphi^{\top}(x, \lambda) \varphi\left(x, \lambda_{n}\right) d x+\sum_{k=2}^{m} \frac{\prod_{i=1}^{k-1} D_{i}}{r_{k}} \int_{c_{k-1}}^{c_{k}} \varphi^{\top}(x, \lambda) \varphi\left(x, \lambda_{n}\right) d x\right. \\
& \left.+\frac{\prod_{i=1}^{m} D_{i}}{r_{m+1}} \int_{c_{m}}^{b} \varphi^{\top}(x, \lambda) \varphi\left(x, \lambda_{n}\right) d x\right) \\
= & \varphi_{1,1}\left(c_{1}^{-}, \lambda\right) \varphi_{2,1}\left(c_{1}^{-}, \lambda_{n}\right)-\varphi_{1,1}\left(c_{1}^{-}, \lambda\right) \varphi_{2,1}\left(c_{1}^{-}, \lambda_{n}\right) \\
& -\left[\varphi_{1,1}(a, \lambda) \varphi_{2,1}\left(a, \lambda_{n}\right)-\varphi_{1,1}(a, \lambda) \varphi_{2,1}\left(a, \lambda_{n}\right)\right] \\
& +\sum_{k=2}^{m} \prod_{i=1}^{k-1} D_{i}\left[\varphi_{1, k}\left(c_{k}^{-}, \lambda\right) \varphi_{2, k}\left(c_{k}^{-}, \lambda_{n}\right)-\varphi_{1, k}\left(c_{k}^{-}, \lambda\right) \varphi_{2, k}\left(c_{k}^{-}, \lambda_{n}\right)\right] \\
& -\sum_{k=2}^{m} \prod_{i=1}^{k-1} D_{i}\left[\varphi_{1, k}\left(c_{k-1}^{+}, \lambda\right) \varphi_{2, k}\left(c_{k-1}^{+}, \lambda_{n}\right)-\varphi_{1, k}\left(c_{k-1}^{+}, \lambda\right) \varphi_{2, k}\left(c_{k-1}^{+}, \lambda_{n}\right)\right] \\
& +\prod_{i=1}^{m} D_{i}\left[\varphi_{1, m+1}(b, \lambda) \varphi_{2, m+1}\left(b, \lambda_{n}\right)-\varphi_{1, m+1}(b, \lambda) \varphi_{2, m+1}\left(b, \lambda_{n}\right)\right] \\
& -\prod_{i=1}^{m} D_{i}\left[\varphi_{1, m+1}\left(c_{m}^{+}, \lambda\right) \varphi_{2, m+1}\left(c_{m}^{+}, \lambda_{n}\right)-\varphi_{1, m+1}\left(c_{m}^{+}, \lambda\right) \varphi_{2, m+1}\left(c_{m}^{+}, \lambda_{n}\right)\right] .
\end{aligned}
$$

Then from the initial conditions (2.16) and (2.20), equation (5.9) becomes

$$
\begin{aligned}
\left(\lambda_{n}-\lambda\right) & \left(\frac{1}{r_{1}} \int_{a}^{c_{1}} \varphi^{\top}(x, \lambda) \varphi\left(x, \lambda_{n}\right) d x+\sum_{k=2}^{m} \frac{\prod_{i=1}^{k-1} D_{i}}{r_{k}} \int_{c_{k-1}}^{c_{k}} \varphi^{\top}(x, \lambda) \varphi\left(x, \lambda_{n}\right) d x\right. \\
& \left.+\frac{\prod_{i=1}^{m} D_{i}}{r_{m+1}} \int_{c_{m}}^{b} \varphi^{\top}(x, \lambda) \varphi\left(x, \lambda_{n}\right) d x\right) \\
= & \prod_{i=1}^{m} D_{i}\left[\varphi_{1, m+1}(b, \lambda) \varphi_{2, m+1}\left(b, \lambda_{n}\right)-\varphi_{1, m+1}(b, \lambda) \varphi_{2, m+1}\left(b, \lambda_{n}\right)\right] .
\end{aligned}
$$

From (2.36) and (2.18), we have

$$
\begin{aligned}
& \varphi_{1, m+1}(b, \lambda) \varphi_{2, m+1}\left(b, \lambda_{n}\right)-\varphi_{2, m+1}(b, \lambda) \varphi_{1, m+1}\left(b, \lambda_{n}\right) \\
& \quad=\tau_{n}^{-1}\left[\varphi_{1, m+1}(b, \lambda) \chi_{2, m+1}\left(b, \lambda_{n}\right)-\varphi_{2, m+1}(b, \lambda) \chi_{1, m+1}\left(b, \lambda_{n}\right)\right] \\
& \quad=\tau_{n}^{-1}\left[-\sin \beta \varphi_{1, m+1}(b, \lambda)-\cos \beta \varphi_{2, m+1}(b, \lambda)\right] \\
& \quad=\tau_{n}^{-1} \omega_{m+1}(\lambda)
\end{aligned}
$$

Substituting from (5.10), (5.11) into (5.8), we get

$$
\left\langle\varphi(\cdot, \lambda), \varphi\left(\cdot, \lambda_{n}\right)\right\rangle_{\mathcal{H}}=\tau_{n}^{-1} \frac{\prod_{i=1}^{m} D_{i} \omega_{m+1}(\lambda)}{\lambda_{n}-\lambda}=\tau_{n}^{-1} \frac{\omega(\lambda)}{\lambda_{n}-\lambda} .
$$

Letting $\lambda \rightarrow \lambda_{n}$ in (5.12) and since the zeros of $\omega(\lambda)$ are simple, we get

$$
\left\langle\varphi\left(\cdot, \lambda_{n}\right), \varphi\left(\cdot, \lambda_{n}\right)\right\rangle_{\mathcal{H}}=\left\|\varphi\left(\cdot, \lambda_{n}\right)\right\|_{\mathcal{H}}^{2}=-\tau_{n}^{-1} \omega^{\prime}\left(\lambda_{n}\right) .
$$


Since $\lambda \in \mathbb{C}$ and $n \in \mathbb{Z}$ are arbitrary, (5.12) and (5.13) hold for all $\lambda \in \mathbb{C}$ and all $n \in \mathbb{Z}$. Therefore from (5.12) and (5.13) we get (5.7). Hence (5.2) is proved with a pointwise convergence on $\mathbb{C}$. Now we investigate the convergence of (5.2). First we prove that it is absolutely convergent on $\mathbb{C}$. Using Cauchy-Schwarz' inequality for $\lambda \in \mathbb{C}$,

$$
\begin{aligned}
& \sum_{k=-\infty}^{\infty}\left|\mathcal{F}\left(\lambda_{k}\right) \frac{\omega(\lambda)}{\left(\lambda-\lambda_{k}\right) \omega^{\prime}\left(\lambda_{k}\right)}\right| \\
& \quad \leq\left(\sum_{k=-\infty}^{\infty} \frac{\left|\left\langle f(\cdot), \varphi\left(\cdot, \lambda_{k}\right)\right\rangle_{\mathcal{H}}\right|^{2}}{\left\|\varphi\left(\cdot, \lambda_{k}\right)\right\|_{\mathcal{H}}^{2}}\right)^{1 / 2}\left(\sum_{k=-\infty}^{\infty} \frac{\left|\left\langle\varphi(\cdot, \lambda), \varphi\left(\cdot, \lambda_{k}\right)\right\rangle_{\mathcal{H}}\right|^{2}}{\left\|\varphi\left(\cdot, \lambda_{k}\right)\right\|_{\mathcal{H}}^{2}}\right)^{1 / 2} .
\end{aligned}
$$

Since $f(\cdot), \varphi(\cdot, \lambda) \in \mathcal{H}$, the two series in the right-hand side of (5.14) converge. Thus series (5.2) converges absolutely on $\mathbb{C}$. As for uniform convergence, let $M \subset \mathbb{C}$ be compact. Let $\lambda \in M$ and $N>0$. Define $v_{N}(\lambda)$ to be

$$
v_{N}(\lambda):=\left|\mathcal{F}(\lambda)-\sum_{k=-N}^{N} \mathcal{F}\left(\lambda_{k}\right) \frac{\omega(\lambda)}{\left(\lambda-\lambda_{k}\right) \omega^{\prime}\left(\lambda_{k}\right)}\right| .
$$

Using the same method as developed above

$$
v_{N}(\lambda) \leq\left(\sum_{k=-N}^{N} \frac{\left|\left\langle f(\cdot), \varphi\left(\cdot, \lambda_{k}\right)\right\rangle_{\mathcal{H}}\right|^{2}}{\left\|\varphi\left(\cdot, \lambda_{k}\right)\right\|_{\mathcal{H}}^{2}}\right)^{1 / 2}\left(\sum_{k=-N}^{N} \frac{\left|\left\langle\varphi(\cdot, \lambda), \varphi\left(\cdot, \lambda_{k}\right)\right\rangle_{\mathcal{H}}\right|^{2}}{\left\|\varphi\left(\cdot, \lambda_{k}\right)\right\|_{\mathcal{H}}^{2}}\right)^{1 / 2} .
$$

Therefore

$$
v_{N}(\lambda) \leq\left\|\varphi\left(\cdot, \lambda_{k}\right)\right\|_{\mathcal{H}}\left(\sum_{k=-N}^{N} \frac{\left|\left\langle f(\cdot), \varphi\left(\cdot, \lambda_{k}\right)\right\rangle_{\mathcal{H}}\right|^{2}}{\left\|\varphi\left(\cdot, \lambda_{k}\right)\right\|_{\mathcal{H}}^{2}}\right)^{1 / 2} .
$$

Since $[a, b] \times M$ is compact, $c f$. e.g. [28], p.225, we can find a positive constant $C_{M}$ such that

$$
\|\varphi(\cdot, \lambda)\|_{\mathcal{H}} \leq C_{M}, \quad \text { for all } \lambda \in M
$$

Then

$$
v_{N}(\lambda) \leq C_{M}\left(\sum_{k=-N}^{N} \frac{\left|\left\langle f(\cdot), \varphi\left(\cdot, \lambda_{k}\right)\right\rangle_{\mathcal{H}}\right|^{2}}{\left\|\varphi\left(\cdot, \lambda_{k}\right)\right\|_{\mathcal{H}}^{2}}\right)^{1 / 2}
$$

uniformly on $M$. In view of Parseval's equality,

$$
\left(\sum_{k=-N}^{N} \frac{\left|\left\langle f(\cdot), \varphi\left(\cdot, \lambda_{k}\right)\right\rangle_{\mathcal{H}}\right|^{2}}{\left\|\varphi\left(\cdot, \lambda_{k}\right)\right\|_{\mathcal{H}}^{2}}\right)^{1 / 2} \rightarrow 0 \quad \text { as } N \rightarrow \infty
$$

Thus $\nu_{N}(\lambda) \rightarrow 0$ uniformly on $M$. Hence (5.2) converges uniformly on $M$. Thus $\mathcal{F}(\lambda)$ is an entire function. From the relation

$$
\begin{aligned}
|\mathcal{F}(\lambda)| \leq & \frac{1}{r_{1}} \int_{a}^{c_{1}}\left|f_{1}(x)\right|\left|\varphi_{1,1}(x, \lambda)\right| d x+\frac{1}{r_{1}} \int_{a}^{c_{1}}\left|f_{2}(x)\right|\left|\varphi_{2,1}(x, \lambda)\right| d x \\
& +\sum_{k=2}^{m} \frac{\prod_{i=1}^{k-1} D_{i}}{r_{k}} \int_{c_{k-1}}^{c_{k}}\left|f_{1}(x)\right|\left|\varphi_{1, k}(x, \lambda)\right| d x
\end{aligned}
$$




$$
\begin{aligned}
& +\sum_{k=2}^{m} \frac{\prod_{i=1}^{k-1} D_{i}}{r_{k}} \int_{c_{k-1}}^{c_{k}}\left|f_{2}(x)\right|\left|\varphi_{2, k}(x, \lambda)\right| d x+\frac{\prod_{i=1}^{m} D_{i}}{r_{m+1}} \int_{c_{m}}^{b}\left|f_{1}(x)\right|\left|\varphi_{1, m+1}(x, \lambda)\right| d x \\
& +\frac{\prod_{i=1}^{m} D_{i}}{r_{m+1}} \int_{c_{m}}^{b}\left|f_{2}(x)\right|\left|\varphi_{2, m+1}(x, \lambda)\right| d x, \quad \lambda \in \mathbb{C},
\end{aligned}
$$

and the fact that $\varphi_{1, k}(\cdot, \lambda)$ and $\varphi_{2, k}(\cdot, \lambda), k=1,2, \ldots, m+1$, are entire functions of exponential type, see Lemma 7.2.1 in [2], we conclude that $\mathcal{F}(\lambda)$ is of exponential type.

Remark 5.2 If we replace $\omega(\lambda)$ by the canonical product

$$
\widetilde{\omega}(\lambda)=\left(\lambda-\lambda_{0}\right) \prod_{n=1}^{\infty}\left(1-\frac{\lambda}{\lambda_{n}}\right)\left(1-\frac{\lambda}{\lambda_{-n}}\right),
$$

then expansion (5.2) is a Lagrange-type interpolation. Indeed, from Hadamard's factorization theorem, see [10], there is an entire function with no zeros, $h(\lambda)$, such that

$$
\omega(\lambda)=h(\lambda) \widetilde{\omega}(\lambda)
$$

Thus,

$$
\frac{\omega(\lambda)}{\omega^{\prime}\left(\lambda_{n}\right)}=\frac{h(\lambda) \widetilde{\omega}(\lambda)}{h\left(\lambda_{n}\right) \widetilde{\omega}^{\prime}\left(\lambda_{n}\right)}
$$

and (5.1), (5.2) remain valid for the function $\mathcal{F}(\lambda) / h(\lambda)$. Hence

$$
\mathcal{F}(\lambda)=\sum_{n=-\infty}^{\infty} \mathcal{F}\left(\lambda_{n}\right) \frac{h(\lambda) \widetilde{\omega}(\lambda)}{h\left(\lambda_{n}\right) \widetilde{\omega}^{\prime}\left(\lambda_{n}\right)\left(\lambda-\lambda_{n}\right)} .
$$

We may redefine (5.1) by taking the kernel $\frac{\varphi(\cdot, \lambda)}{h(\lambda)}=\widetilde{\varphi}(\cdot, \lambda)$ to get

$$
\widetilde{\mathcal{F}}(\lambda)=\frac{\mathcal{F}(\lambda)}{h(\lambda)}=\sum_{n=-\infty}^{\infty} \widetilde{\mathcal{F}}\left(\lambda_{n}\right) \frac{\widetilde{\omega}(\lambda)}{\left(\lambda-\lambda_{n}\right) \widetilde{\omega}^{\prime}\left(\lambda_{n}\right)} .
$$

For more details as regards the argument of Remark 5.2, see [9], p.110.

The next theorem is devoted to vector-type interpolation sampling expansions associated with problem (2.1)-(2.4) for vector-valued integral transforms whose kernels are defined in terms of the Green's matrix. As we see in (4.16), the Green's matrix $G(x, \xi, \lambda)$ of problem (2.1)-(2.4) has simple poles at $\left\{\lambda_{k}\right\}_{k=-\infty}^{\infty}$. Define the function $G(x, \lambda)$ to be $G(x, \lambda):=\omega(\lambda) G\left(x, \xi_{0}, \lambda\right)$, where $\xi_{0} \in\left[a, c_{1}\right) \cup\left(c_{1}, c_{2}\right) \cup\left(c_{2}, c_{3}\right) \cup \cdots \cup\left(c_{m}, b\right]$ is a fixed point and $\omega(\lambda)$ is the function defined in (2.24) or it is the canonical product (5.20).

Theorem 5.3 Let $f(x)=\left(\begin{array}{c}f_{1}(x) \\ f_{2}(x)\end{array}\right) \in \mathcal{H}$. Let $\mathfrak{F}(\lambda)=\left(\begin{array}{c}\mathfrak{F}_{1}(\lambda) \\ \mathfrak{F}_{2}(\lambda)\end{array}\right)$ be the vector-valued transform

$$
\begin{aligned}
\mathfrak{F}(\lambda)= & \frac{1}{r_{1}} \int_{a}^{c_{1}} G(x, \lambda) f(x) d x+\sum_{k=2}^{m} \frac{\prod_{i=1}^{k-1} D_{i}}{r_{k}} \int_{c_{k-1}}^{c_{k}} G(x, \lambda) f(x) d x \\
& +\frac{\prod_{i=1}^{m} D_{i}}{r_{m+1}} \int_{c_{m}}^{b} G(x, \lambda) f(x) d x .
\end{aligned}
$$


Then $\mathfrak{F}(\lambda)$ is a vector-valued entire function of exponential type that admits the vectorvalued sampling expansion

$$
\mathfrak{F}(\lambda)=\sum_{n=-\infty}^{\infty} \mathfrak{F}\left(\lambda_{n}\right) \frac{\omega(\lambda)}{\left(\lambda-\lambda_{n}\right) \omega^{\prime}\left(\lambda_{n}\right)}
$$

The vector-valued series (5.24) converges absolutely on $\mathbb{C}$ and uniformly on compact subsets of $\mathbb{C}$. Here (5.24) means

$$
\begin{aligned}
& \mathfrak{F}_{1}(\lambda)=\sum_{n=-\infty}^{\infty} \mathfrak{F}_{1}\left(\lambda_{n}\right) \frac{\omega(\lambda)}{\left(\lambda-\lambda_{n}\right) \omega^{\prime}\left(\lambda_{n}\right)}, \\
& \mathfrak{F}_{2}(\lambda)=\sum_{n=-\infty}^{\infty} \mathfrak{F}_{2}\left(\lambda_{n}\right) \frac{\omega(\lambda)}{\left(\lambda-\lambda_{n}\right) \omega^{\prime}\left(\lambda_{n}\right)},
\end{aligned}
$$

where both series converge absolutely on $\mathbb{C}$ and uniformly on compact sets of $\mathbb{C}$.

Proof Let $\lambda \in \mathbb{C}$ such that $\lambda \neq \lambda_{n}$ for $n \in \mathbb{Z}$. Since $\mathfrak{F}(\lambda)$ is nothing but the unique solution (multiplied by $\omega(\lambda)$ ) of the inhomogeneous Dirac system (4.1), (2.2)-(2.4) when $\xi=\xi_{0}$, see [1], p.77, $\mathfrak{F}(\lambda)$ has the expansion

$$
\begin{aligned}
\mathfrak{F}(\lambda) & =\sum_{n=-\infty}^{\infty} \frac{a_{n} \omega(\lambda)}{\lambda_{n}-\lambda} \psi_{n}\left(\xi_{0}\right), \\
a_{n}= & \frac{1}{r_{1}} \int_{a}^{c_{1}} f^{\top}(x) \psi_{n}(x) d x+\sum_{k=2}^{m} \frac{\prod_{i=1}^{k-1} D_{i}}{r_{k}} \int_{c_{k-1}}^{c_{k}} f^{\top}(x) \psi_{n}(x) d x \\
& +\frac{\prod_{i=1}^{m} D_{i}}{r_{m+1}} \int_{c_{m}}^{b} f^{\top}(x) \psi_{n}(x) d x
\end{aligned}
$$

and $\left\{\psi_{n}(\cdot)\right\}_{n=-\infty}^{\infty}$ is a complete orthonormal set of vector-valued eigenfunctions. Thus

$$
\mathfrak{F}(\lambda)=\sum_{n=-\infty}^{\infty} \frac{a_{n} \omega(\lambda)}{\lambda_{n}-\lambda} \frac{\varphi\left(\xi_{0}, \lambda_{n}\right)}{\left\|\varphi\left(\cdot, \lambda_{n}\right)\right\|_{\mathcal{H}}^{2}}
$$

Now, we calculate $\left\|\varphi\left(\cdot, \lambda_{n}\right)\right\|_{\mathcal{H}}^{2}$ using the Green's identity [1], p. 51. Let $\lambda, \mu \in \mathbb{C}$ be different and $\mu \neq \lambda_{n} \neq \lambda$; then, from (2.20) and (2.22),

$$
\begin{aligned}
(\lambda-\mu) & \left(\frac{1}{r_{1}} \int_{a}^{c_{1}} \varphi^{\top}(x, \lambda) \bar{\chi}(x, \mu) d x+\sum_{k=2}^{m} \frac{\prod_{i=1}^{k-1} D_{i}}{r_{k}} \int_{c_{k-1}}^{c_{k}} \varphi^{\top}(x, \lambda) \bar{\chi}(x, \mu) d x\right. \\
& \left.+\frac{\prod_{i=1}^{m} D_{i}}{r_{m+1}} \int_{c_{m}}^{b} \varphi^{\top}(x, \lambda) \bar{\chi}(x, \mu) d x\right) \\
= & \prod_{i=1}^{m} D_{i}\left[\varphi_{1, m+1}(b, \lambda) \bar{\chi}_{2, m+1}(b, \mu)-\varphi_{2, m+1}(b, \lambda) \bar{\chi}_{1, m+1}(b, \mu)\right] \\
& -\left(\varphi_{1,1}(a, \lambda) \bar{\chi}_{2,1}(a, \mu)-\varphi_{2,1}(a, \lambda) \bar{\chi}_{1,1}(a, \mu)\right),
\end{aligned}
$$


where $\varphi(x, \lambda)$ and $\chi(x, \mu)$ are the solutions defined above. By the initial conditions (2.16) and (2.18), we have

$$
\begin{aligned}
(\lambda-\mu) & \left(\frac{1}{r_{1}} \int_{a}^{c_{1}} \varphi^{\top}(x, \lambda) \bar{\chi}(x, \mu) d x+\sum_{k=2}^{m} \frac{\prod_{i=1}^{k-1} D_{i}}{r_{k}} \int_{c_{k-1}}^{c_{k}} \varphi^{\top}(x, \lambda) \bar{\chi}(x, \mu) d x\right. \\
& \left.+\frac{\prod_{i=1}^{m} D_{i}}{r_{m+1}} \int_{c_{m}}^{b} \varphi^{\top}(x, \lambda) \bar{\chi}(x, \mu) d x\right) \\
= & -\prod_{i=1}^{m} D_{i}\left(\sin \beta \varphi_{1, m+1}(b, \lambda)+\cos \beta \varphi_{2, m+1}(b, \lambda)\right) \\
& -\left(\cos \alpha \bar{\chi}_{2,1}(a, \mu)+\sin \alpha \bar{\chi}_{1,1}(a, \mu)\right) .
\end{aligned}
$$

Letting $\mu=\lambda_{n}$, for some $n$, and noting that $\chi\left(x, \lambda_{n}\right)$ is a real-valued eigenfunction, from (2.16) and (2.36), equation (5.29) becomes

$$
\begin{aligned}
\tau_{n}(\lambda & \left.-\lambda_{n}\right)\left(\frac{1}{r_{1}} \int_{a}^{c_{1}} \varphi^{\top}(x, \lambda) \varphi\left(x, \lambda_{n}\right) d x+\sum_{k=2}^{m} \frac{\prod_{i=1}^{k-1} D_{i}}{r_{k}} \int_{c_{k-1}}^{c_{k}} \varphi^{\top}(x, \lambda) \varphi\left(x, \lambda_{n}\right) d x\right. \\
& \left.+\frac{\prod_{i=1}^{m} D_{i}}{r_{m+1}} \int_{c_{m}}^{b} \varphi^{\top}(x, \lambda) \varphi\left(x, \lambda_{n}\right) d x\right) \\
= & -\prod_{i=1}^{m} D_{i}\left(\sin \beta \varphi_{1, m+1}(b, \lambda)+\cos \beta \varphi_{2, m+1}(b, \lambda)\right) .
\end{aligned}
$$

Then from (2.24), we obtain

$$
\begin{aligned}
& \frac{1}{r_{1}} \int_{a}^{c_{1}} \varphi^{\top}(x, \lambda) \varphi\left(x, \lambda_{n}\right) d x+\sum_{k=2}^{m} \frac{\prod_{i=1}^{k-1} D_{i}}{r_{k}} \int_{c_{k-1}}^{c_{k}} \varphi^{\top}(x, \lambda) \varphi\left(x, \lambda_{n}\right) d x \\
& \quad+\frac{\prod_{i=1}^{m} D_{i}}{r_{m+1}} \int_{c_{m}}^{b} \varphi^{\top}(x, \lambda) \varphi\left(x, \lambda_{n}\right) d x=\frac{\tau_{n}^{-1} \omega(\lambda)}{\lambda-\lambda_{n}} .
\end{aligned}
$$

Taking the limit in (5.31) when $\lambda \rightarrow \lambda_{n}$,

$$
\begin{aligned}
\left\|\varphi\left(\cdot, \lambda_{n}\right)\right\|_{\mathcal{H}}^{2}= & \frac{1}{r_{1}} \int_{a}^{c_{1}} \varphi^{\top}(x, \lambda) \varphi\left(x, \lambda_{n}\right) d x+\sum_{k=2}^{m} \frac{\prod_{i=1}^{k-1} D_{i}}{r_{k}} \int_{c_{k-1}}^{c_{k}} \varphi^{\top}(x, \lambda) \varphi\left(x, \lambda_{n}\right) d x \\
& +\frac{\prod_{i=1}^{m} D_{i}}{r_{m+1}} \int_{c_{m}}^{b} \varphi^{\top}(x, \lambda) \varphi\left(x, \lambda_{n}\right) d x=\tau_{n}^{-1} \omega^{\prime}\left(\lambda_{n}\right) .
\end{aligned}
$$

Substituting in (5.27), we get the following series, which holds if $\lambda$ is not an eigenvalue:

$$
\mathfrak{F}(\lambda)=\sum_{n=-\infty}^{\infty} \frac{a_{n} \tau_{n} \omega(\lambda)}{\lambda_{n}-\lambda} \frac{\varphi\left(\xi_{0}, \lambda_{n}\right)}{\omega^{\prime}\left(\lambda_{n}\right)}
$$

To prove (5.24), it suffices to prove that $\mathfrak{F}\left(\lambda_{n}\right)=a_{n} \tau_{n} \varphi\left(\xi_{0}, \lambda_{n}\right)$. Indeed, from (4.16) and (5.23), we have

$$
\mathfrak{F}\left(\lambda_{n}\right)=\left(\begin{array}{l}
\mathfrak{F}_{1}\left(\lambda_{n}\right) \\
\mathfrak{F}_{2}\left(\lambda_{n}\right)
\end{array}\right),
$$


where

$$
\mathfrak{F}_{1}\left(\lambda_{n}\right)=\left\{\begin{array}{l}
\frac{\varphi_{1}\left(\xi_{0}, \lambda_{n}\right)}{r_{1}} \int_{\xi_{0}}^{c_{1}} \chi^{\top}\left(x, \lambda_{n}\right) f(x) d x+\frac{\chi_{1}\left(\xi_{0}, \lambda_{n}\right)}{r_{1}} \int_{a}^{\xi_{0}} \varphi^{\top}\left(x, \lambda_{n}\right) f(x) d x \\
\quad+\sum_{i=1}^{m} \frac{\varphi_{1}\left(\xi_{0}, \lambda_{n}\right) \prod_{j=1}^{i} D_{j}}{r_{i+1}} \int_{c_{i}}^{c_{i+1}} \chi^{\top}\left(x, \lambda_{n}\right) f(x) d x, \quad \xi_{0} \in\left[a, c_{1}\right), \\
\quad+\frac{\varphi_{1}\left(\xi_{0}, \lambda_{n}\right) \prod_{i=1}^{k-1} D_{i}}{r_{k}} \int_{\xi_{0}}^{c_{k}} \chi^{\top}\left(x, \lambda_{n}\right) f(x) d x \\
\quad+\frac{\chi_{1}\left(\xi_{0}, \lambda_{n}\right) \prod_{i=1}^{k-1} D_{i}}{r_{k}} \int_{c_{k-1}}^{\xi_{0}} \varphi^{\top}\left(x, \lambda_{n}\right) f(x) d x \\
\quad+\sum_{i=k}^{m} \frac{\varphi_{1}\left(\xi_{0}, \lambda_{n}\right) \prod_{j=1}^{i} D_{j}}{r_{i+1}} \int_{c_{i}}^{c_{i+1}} \chi^{\top}\left(x, \lambda_{n}\right) f(x) d x \\
\quad+\sum_{i=1}^{k-1} \frac{\chi_{1}\left(\xi_{0}, \lambda_{n}\right) \prod_{j=1}^{i} D_{j-1}}{r_{i}} \int_{c_{i-1}}^{c_{i}} \varphi^{\top}\left(x, \lambda_{n}\right) f(x) d x, \\
\quad \xi_{0} \in\left(c_{k-1}, c_{k}\right)(k=2,3, \ldots, m), D_{0}=1, \\
\frac{\varphi_{1}\left(\xi_{0}, \lambda_{n}\right) \prod_{i=1}^{m} D_{i}}{r_{m+1}} \int_{\xi_{0}}^{b} \chi^{\top}\left(x, \lambda_{n}\right) f(x) d x \\
\quad+\frac{\chi_{1}\left(\xi_{0}, \lambda_{n}\right) \prod_{i=1}^{m} D_{i}}{r_{m+1}} \int_{c_{m}}^{\xi_{0}} \varphi^{\top}\left(x, \lambda_{n}\right) f(x) d x \\
\quad+\sum_{i=1}^{m} \frac{\chi_{1}\left(\xi_{0}, \lambda_{n}\right) \prod_{j=1}^{i} D_{j-1}}{r_{i}} \int_{c_{i-1}}^{c_{i}} \varphi^{\top}\left(x, \lambda_{n}\right) f(x) d x, \quad \xi_{0} \in\left(c_{m}, b\right]
\end{array}\right.
$$

and

$$
\mathfrak{F}_{2}\left(\lambda_{n}\right)=\left\{\begin{array}{l}
\frac{\varphi_{2}\left(\xi_{0}, \lambda_{n}\right)}{r_{1}} \int_{\xi_{0}}^{c_{1}} \chi^{\top}\left(x, \lambda_{n}\right) f(x) d x+\frac{\chi_{2}\left(\xi_{0}, \lambda_{n}\right)}{r_{1}} \int_{a}^{\xi_{0}} \varphi^{\top}\left(x, \lambda_{n}\right) f(x) d x \\
\quad+\sum_{i=1}^{m} \frac{\varphi_{2}\left(\xi_{0}, \lambda_{n}\right) \prod_{j=1}^{i} D_{j}}{r_{i+1}} \int_{c_{i}}^{c_{i+1}} \chi^{\top}\left(x, \lambda_{n}\right) f(x) d x, \quad \xi_{0} \in\left[a, c_{1}\right), \\
\frac{\varphi_{2}\left(\xi_{0}, \lambda_{n}\right) \prod_{i=1}^{k-1} D_{i}}{r_{k}} \int_{\xi_{0}}^{c_{k}} \chi^{\top}\left(x, \lambda_{n}\right) f(x) d x \\
\quad+\frac{\chi_{2}\left(\xi_{0}, \lambda_{n}\right) \prod_{i=1}^{k-1} D_{i}}{r_{k}} \int_{c_{k-1}}^{\xi_{0}} \varphi^{\top}\left(x, \lambda_{n}\right) f(x) d x \\
\quad+\sum_{i=k}^{m} \frac{\varphi_{2}\left(\xi_{0}, \lambda_{n}\right) \prod_{j=1}^{i} D_{j}}{r_{i+1}} \int_{c_{i}}^{c_{i+1}} \chi^{\top}\left(x, \lambda_{n}\right) f(x) d x \\
\quad+\sum_{i=1}^{k-1} \frac{\chi_{2}\left(\xi_{0}, \lambda_{n}\right) \prod_{j=1}^{i} D_{j-1}}{r_{i}} \int_{c_{i-1}}^{c_{i}} \varphi^{\top}\left(x, \lambda_{n}\right) f(x) d x, \\
\xi_{0} \in\left(c_{k-1}, c_{k}\right)(k=2,3, \ldots, m), D_{0}=1, \\
\frac{\varphi_{2}\left(\xi_{0}, \lambda_{n}\right) \prod_{i=1}^{m} D_{i}}{r_{m+1}} \int_{\xi_{0}}^{b} \chi^{\top}\left(x, \lambda_{n}\right) f(x) d x \\
\quad+\frac{\chi_{2}\left(\xi_{0}, \lambda_{n}\right) \prod_{i=1}^{m} D_{i}}{r_{m+1}} \int_{c_{m}}^{\xi_{0}} \varphi^{\top}\left(x, \lambda_{n}\right) f(x) d x \\
\quad+\sum_{i=1}^{m} \frac{\chi_{2}\left(\xi_{0}, \lambda_{n}\right) \prod_{j=1}^{i} D_{j-1}}{r_{i}} \int_{c_{i-1}}^{c_{i}} \varphi^{\top}\left(x, \lambda_{n}\right) f(x) d x, \quad \xi_{0} \in\left(c_{m}, b\right] .
\end{array}\right.
$$

Now we prove that $\mathfrak{F}_{1}\left(\lambda_{n}\right)=a_{n} \tau_{n} \varphi_{1}\left(\xi_{0}, \lambda_{n}\right)$ and $\mathfrak{F}_{2}\left(\lambda_{n}\right)=a_{n} \tau_{n} \varphi_{2}\left(\xi_{0}, \lambda_{n}\right)$. From (2.36), (5.35), and (5.36), we get

$$
\begin{aligned}
\mathfrak{F}_{1}\left(\lambda_{n}\right)= & \tau_{n} \varphi_{1}\left(\xi_{0}, \lambda_{n}\right)\left(\frac{1}{r_{1}} \int_{a}^{c_{1}} \varphi^{\top}\left(x, \lambda_{n}\right) f(x) d x\right. \\
& +\sum_{k=2}^{m} \frac{\prod_{i=1}^{k-1} D_{i}}{r_{k}} \int_{c_{k-1}}^{c_{k}} \varphi^{\top}\left(x, \lambda_{n}\right) f(x) d x \\
& \left.+\frac{\prod_{i=1}^{m} D_{i}}{r_{m+1}} \int_{c_{m}}^{b} \varphi^{\top}\left(x, \lambda_{n}\right) f(x) d x\right) \\
= & \tau_{n} \varphi_{1}\left(\xi_{0}, \lambda_{n}\right)\left(\frac{1}{r_{1}} \int_{a}^{c_{1}} f^{\top}(x) \varphi\left(x, \lambda_{n}\right) d x\right. \\
& +\sum_{k=2}^{m} \frac{\prod_{i=1}^{k-1} D_{i}}{r_{k}} \int_{c_{k-1}}^{c_{k}} f^{\top}(x) \varphi\left(x, \lambda_{n}\right) d x
\end{aligned}
$$




$$
\begin{aligned}
& \left.+\frac{\prod_{i=1}^{m} D_{i}}{r_{m+1}} \int_{c_{m}}^{b} f^{\top}(x) \varphi\left(x, \lambda_{n}\right) d x\right) \\
= & a_{n} \tau_{n} \varphi_{1}\left(\xi_{0}, \lambda_{n}\right),
\end{aligned}
$$

for $x, \xi_{0} \in\left[a, c_{1}\right) \cup\left(c_{1}, c_{2}\right) \cup\left(c_{2}, c_{3}\right) \cup \cdots \cup\left(c_{m}, b\right]$. The same for $\mathfrak{F}_{2}\left(\lambda_{n}\right)$ and the proof of (5.24) is complete. The convergence properties as well as the analytic and growth properties can be established as in Theorem 5.1 above.

\section{Examples}

Example 6.1 The boundary value problem

$$
\begin{aligned}
& u_{2}^{\prime}-q(x) u_{1}=\lambda u_{1}, \quad u_{1}^{\prime}+q(x) u_{2}=-\lambda u_{2}, \quad x \in[-1,0) \cup\left(0, \frac{1}{2}\right) \cup\left(\frac{1}{2}, 1\right], \\
& u_{1}(-1)=u_{1}(1)=0, \\
& u_{1}\left(0^{-}\right)-2 u_{1}\left(0^{+}\right)=0, \quad u_{2}\left(0^{-}\right)-2 u_{2}\left(0^{+}\right)=0, \\
& 2 u_{1}\left(\frac{1}{2}^{-}\right)-u_{1}\left(\frac{1}{2}^{+}\right)=0, \quad 2 u_{2}\left(\frac{1}{2}^{-}\right)-u_{2}\left(\frac{1}{2}^{+}\right)=0,
\end{aligned}
$$

is a special case of problem (2.1)-(2.4) when $m=2, r_{1}=r_{2}=r_{3}=1, \gamma_{1}=\gamma_{1}^{\prime}=\delta_{2}=\delta_{2}^{\prime}=1$, $\gamma_{2}=\gamma_{2}^{\prime}=\delta_{1}=\delta_{1}^{\prime}=2, \alpha=\beta=\frac{\pi}{2}$ and $q_{1}(x)=q_{2}(x)=q(x)$,

$$
q(x)= \begin{cases}-1, & -1 \leq x<0 \\ 0, & 0<x<\frac{1}{2} \\ 1, & \frac{1}{2}<x \leq 1\end{cases}
$$

Then $\gamma_{k} \delta_{k}^{\prime}=\gamma_{k}^{\prime} \delta_{k}, k=1,2$. In the notations of Section 2 , the solutions $\varphi(\cdot, \lambda)$ and $\chi(\cdot, \lambda)$ are

$$
\begin{aligned}
& \varphi(x, \lambda)= \begin{cases}{\left[\begin{array}{l}
\varphi_{1,1}(x, \lambda) \\
\varphi_{2,1}(x, \lambda)
\end{array}\right]=\left[\begin{array}{c}
\sin [(\lambda-1)(x+1)] \\
-\cos [(\lambda-1)(x+1)]
\end{array}\right],} & x \in[-1,0), \\
{\left[\begin{array}{l}
\varphi_{1,2}(x, \lambda) \\
\varphi_{2,2}(x, \lambda)
\end{array}\right]=\left[\begin{array}{c}
-\frac{1}{2} \sin [1-\lambda(x+1)] \\
-\frac{1}{2} \cos [1-\lambda(x+1)]
\end{array}\right],} & x \in\left(0, \frac{1}{2}\right), \\
{\left[\begin{array}{l}
\varphi_{1,3}(x, \lambda) \\
\varphi_{2,3}(x, \lambda)
\end{array}\right]=\left[\begin{array}{ll}
-\sin \left[\frac{3}{2}-\lambda-(\lambda+1) x\right] \\
-\cos \left[\frac{3}{2}-\lambda-(\lambda+1) x\right]
\end{array}\right],} & x \in\left(\frac{1}{2}, 1\right],\end{cases} \\
& \chi(x, \lambda)= \begin{cases}{\left[\begin{array}{l}
\chi_{1,1}(x, \lambda) \\
\chi_{2,1}(x, \lambda)
\end{array}\right]=\left[\begin{array}{c}
-\sin \left[\lambda+\frac{1}{2}+(1-\lambda) x\right] \\
-\cos \left[\lambda+\frac{1}{2}+(1-\lambda) x\right]
\end{array}\right],} & x \in[-1,0), \\
{\left[\begin{array}{l}
\chi_{1,2}(x, \lambda) \\
\chi_{2,2}(x, \lambda)
\end{array}\right]=\left[\begin{array}{c}
-\frac{1}{2} \sin \left[\lambda+\frac{1}{2}-\lambda x\right] \\
-\frac{1}{2} \cos \left[\lambda+\frac{1}{2}-\lambda x\right]
\end{array}\right],} & x \in\left(0, \frac{1}{2}\right), \\
{\left[\begin{array}{l}
\chi_{1,3}(x, \lambda) \\
\chi_{2,3}(x, \lambda)
\end{array}\right]=\left[\begin{array}{c}
\sin [(\lambda+1)(x-1)] \\
-\cos [(\lambda+1)(x-1)]
\end{array}\right],} & x \in\left(\frac{1}{2}, 1\right] .\end{cases}
\end{aligned}
$$

The eigenvalues are the solutions of the equation

$$
\omega(\lambda)=\sin \left[\frac{1}{2}-2 \lambda\right]=0 .
$$

Hence the eigenvalues are

$$
\lambda_{n}=\frac{1-2 n \pi}{4}, \quad n \in \mathbb{Z},
$$




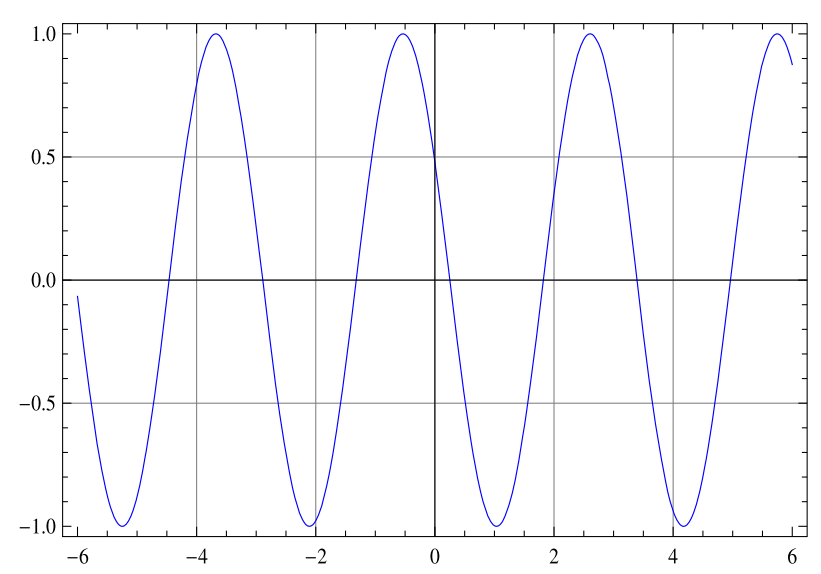

Figure 1 The eigenvalues of $\omega(\lambda)$.

which is illustrated in Figure 1. The Green's matrix of problem (6.1)-(6.4) is given by

$$
G(x, \xi, \lambda)=\frac{1}{\sin \left[\frac{1}{2}-2 \lambda\right]} \begin{cases}G_{1}(x, \xi, \lambda), & -1 \leq \xi \leq x<0, \\ G_{2}(x, \xi, \lambda), & -1 \leq x \leq \xi<0, \\ G_{3}(x, \xi, \lambda), & -1 \leq \xi<0,0<x<\frac{1}{2}, \\ G_{4}(x, \xi, \lambda), & -1 \leq x<0,0<\xi<\frac{1}{2}, \\ G_{5}(x, \xi, \lambda), & -1 \leq \xi<0, \frac{1}{2}<x \leq 1, \\ G_{6}(x, \xi, \lambda), & -1 \leq x<0, \frac{1}{2}<\xi \leq 1, \\ G_{7}(x, \xi, \lambda), & 0<\xi \leq x<\frac{1}{2}, \\ G_{8}(x, \xi, \lambda), & 0<x \leq \xi<\frac{1}{2}, \\ G_{9}(x, \xi, \lambda), & 0<\xi<\frac{1}{2}, \frac{1}{2}<x \leq 1, \\ G_{10}(x, \xi, \lambda), & 0<x<\frac{1}{2}, \frac{1}{2}<\xi \leq 1, \\ G_{11}(x, \xi, \lambda), & \frac{1}{2}<\xi \leq x \leq 1, \\ G_{12}(x, \xi, \lambda), & \frac{1}{2}<x \leq \xi \leq 1,\end{cases}
$$

where

$G_{1}(x, \xi, \lambda)$

$=\left(\begin{array}{ll}-\sin [(\lambda-1)(\xi+1)] \sin \left[\lambda+\frac{1}{2}+(1-\lambda) x\right] & \cos [(\lambda-1)(\xi+1)] \sin \left[\lambda+\frac{1}{2}+(1-\lambda) x\right] \\ -\sin [(\lambda-1)(\xi+1)] \cos \left[\lambda+\frac{1}{2}+(1-\lambda) x\right] & \cos [(\lambda-1)(\xi+1)] \cos \left[\lambda+\frac{1}{2}+(1-\lambda) x\right]\end{array}\right)$,

$G_{2}(x, \xi, \lambda)$

$=\left(\begin{array}{cc}-\sin [(\lambda-1)(x+1)] \sin \left[\lambda+\frac{1}{2}+(1-\lambda) \xi\right] & -\sin [(\lambda-1)(x+1)] \cos \left[\lambda+\frac{1}{2}+(1-\lambda) \xi\right] \\ \cos [(\lambda-1)(x+1)] \sin \left[\lambda+\frac{1}{2}+(1-\lambda) \xi\right] & \cos [(\lambda-1)(x+1)] \cos \left[\lambda+\frac{1}{2}+(1-\lambda) \xi\right]\end{array}\right)$,

$G_{3}(x, \xi, \lambda)$

$=\frac{1}{2}\left(\begin{array}{ll}-\sin [(\lambda-1)(\xi+1)] \sin \left[\lambda+\frac{1}{2}-\lambda x\right] & \cos [(\lambda-1)(\xi+1)] \sin \left[\lambda+\frac{1}{2}-\lambda x\right] \\ -\sin [(\lambda-1)(\xi+1)] \cos \left[\lambda+\frac{1}{2}-\lambda x\right] & \cos [(\lambda-1)(\xi+1)] \cos \left[\lambda+\frac{1}{2}-\lambda x\right]\end{array}\right)$,

$G_{4}(x, \xi, \lambda)$

$=\frac{1}{2}\left(\begin{array}{cc}-\sin [(\lambda-1)(x+1)] \sin \left[\lambda+\frac{1}{2}-\lambda \xi\right] & -\sin [(\lambda-1)(x+1)] \cos \left[\lambda+\frac{1}{2}-\lambda \xi\right] \\ \cos [(\lambda-1)(x+1)] \sin \left[\lambda+\frac{1}{2}-\lambda \xi\right] & \cos [(\lambda-1)(x+1)] \cos \left[\lambda+\frac{1}{2}-\lambda \xi\right]\end{array}\right)$, 
$G_{5}(x, \xi, \lambda)$

$$
=\left(\begin{array}{cc}
\sin [(\lambda-1)(\xi+1)] \sin [(\lambda+1)(x-1)] & -\cos [(\lambda-1)(\xi+1)] \sin [(\lambda+1)(x-1)] \\
-\sin [(\lambda-1)(\xi+1)] \cos [(\lambda+1)(x-1)] & \cos [(\lambda-1)(\xi+1)] \cos [(\lambda+1)(x-1)]
\end{array}\right),
$$

$G_{6}(x, \xi, \lambda)$

$$
=\left(\begin{array}{cc}
\sin [(\lambda-1)(x+1)] \sin [(\lambda+1)(\xi-1)] & -\sin [(\lambda-1)(x+1)] \cos [(\lambda+1)(\xi-1)] \\
-\cos [(\lambda-1)(x+1)] \sin [(\lambda+1)(\xi-1)] & \cos [(\lambda-1)(x+1)] \cos [(\lambda+1)(\xi-1)]
\end{array}\right),
$$

$G_{7}(x, \xi, \lambda)$

$$
=\frac{1}{4}\left(\begin{array}{cc}
\sin [1-\lambda(\xi+1)] \sin \left[\lambda+\frac{1}{2}-\lambda x\right] & \cos [1-\lambda(\xi+1)] \sin \left[\lambda+\frac{1}{2}-\lambda x\right] \\
\sin [1-\lambda(\xi+1)] \cos \left[\lambda+\frac{1}{2}-\lambda x\right] & \cos [1-\lambda(\xi+1)] \cos \left[\lambda+\frac{1}{2}-\lambda x\right]
\end{array}\right),
$$

$G_{8}(x, \xi, \lambda)$

$$
=\frac{1}{4}\left(\begin{array}{ll}
\sin [1-\lambda(x+1)] \sin \left[\lambda+\frac{1}{2}-\lambda \xi\right] & \sin [1-\lambda(x+1)] \cos \left[\lambda+\frac{1}{2}-\lambda \xi\right] \\
\cos [1-\lambda(x+1)] \sin \left[\lambda+\frac{1}{2}-\lambda \xi\right] & \cos [1-\lambda(x+1)] \cos \left[\lambda+\frac{1}{2}-\lambda \xi\right]
\end{array}\right),
$$

$G_{9}(x, \xi, \lambda)$

$$
=\frac{1}{2}\left(\begin{array}{cc}
-\sin [1-\lambda(\xi+1)] \sin [(\lambda+1)(x-1)] & -\cos [1-\lambda(\xi+1)] \sin [(\lambda+1)(x-1)] \\
\sin [1-\lambda(\xi+1)] \cos [(\lambda+1)(x-1)] & \cos [1-\lambda(\xi+1)] \cos [(\lambda+1)(x-1)]
\end{array}\right),
$$

$G_{10}(x, \xi, \lambda)$

$$
=\frac{1}{2}\left(\begin{array}{ll}
-\sin [1-\lambda(x+1)] \sin [(\lambda+1)(\xi-1)] & \sin [1-\lambda(x+1)] \cos [(\lambda+1)(\xi-1)] \\
-\cos [1-\lambda(x+1)] \sin [(\lambda+1)(\xi-1)] & \cos [1-\lambda(x+1)] \cos [(\lambda+1)(\xi-1)]
\end{array}\right),
$$

$G_{11}(x, \xi, \lambda)$

$$
=\left(\begin{array}{cc}
-\sin \left[\frac{3}{2}-\lambda-(\lambda+1) \xi\right] \sin [(\lambda+1)(x-1)] & -\cos \left[\frac{3}{2}-\lambda-(\lambda+1) \xi\right] \sin [(\lambda+1)(x-1)] \\
\sin \left[\frac{3}{2}-\lambda-(\lambda+1) \xi\right] \cos [(\lambda+1)(x-1)] & \cos \left[\frac{3}{2}-\lambda-(\lambda+1) \xi\right] \cos [(\lambda+1)(x-1)]
\end{array}\right),
$$

$G_{12}(x, \xi, \lambda)$

$$
=\left(\begin{array}{ll}
-\sin \left[\frac{3}{2}-\lambda-(\lambda+1) x\right] \sin [(\lambda+1)(\xi-1)] & \sin \left[\frac{3}{2}-\lambda-(\lambda+1) x\right] \cos [(\lambda+1)(\xi-1)] \\
-\cos \left[\frac{3}{2}-\lambda-(\lambda+1) x\right] \sin [(\lambda+1)(\xi-1)] & \cos \left[\frac{3}{2}-\lambda-(\lambda+1) x\right] \cos [(\lambda+1)(\xi-1)]
\end{array}\right) .
$$

By Theorem 5.1, the transform

$$
\begin{aligned}
\mathcal{F}(\lambda)= & \int_{-1}^{0}\left[f_{1}(x) \sin [(\lambda-1)(x+1)]-f_{2}(x) \cos [(\lambda-1)(x+1)]\right] d x \\
& -2 \int_{0}^{\frac{1}{2}}\left[f_{1}(x) \sin [1-\lambda(x+1)]+f_{2}(x) \cos [1-\lambda(x+1)]\right] d x \\
& -\int_{\frac{1}{2}}^{1}\left[f_{1}(x) \sin \left[\frac{3}{2}-\lambda-(\lambda+1) x\right]+f_{2}(x) \cos \left[\frac{3}{2}-\lambda-(\lambda+1) x\right]\right] d x
\end{aligned}
$$

has the following expansion:

$$
\mathcal{F}(\lambda)=\sum_{n=-\infty}^{\infty} \mathcal{F}\left(\frac{1-2 n \pi}{4}\right) \frac{\sin \left[\frac{1}{2}+n \pi-2 \lambda\right]}{\frac{1}{2}-n \pi-2 \lambda}
$$

where $\omega^{\prime}\left(\frac{1-2 n \pi}{4}\right)=2(-1)^{n+1}$. 
In view of Theorem 5.3, the vector-valued transform

$$
\mathfrak{F}(\lambda)= \begin{cases}{\left[\begin{array}{c}
-\sin \left[\lambda+\frac{1}{2}+(1-\lambda) \xi_{0}\right] \\
-\cos \left[\lambda+\frac{1}{2}+(1-\lambda) \xi_{0}\right]
\end{array}\right] \mathcal{T}_{1}+\left[\begin{array}{c}
\sin \left[(\lambda-1)\left(\xi_{0}+1\right)\right] \\
-\cos \left[(\lambda-1)\left(\xi_{0}+1\right)\right]
\end{array}\right]\left(\mathcal{T}_{2}+\mathcal{T}_{3}+\mathcal{T}_{4}\right),} & -1 \leq \xi_{0}<0, \\
\frac{1}{2}\left[\begin{array}{c}
-\sin \left[\lambda+\frac{1}{2}-\lambda \xi_{0}\right] \\
-\cos \left[\lambda+\frac{1}{2}-\lambda \xi_{0}\right]
\end{array}\right]\left(\mathcal{T}_{5}+\mathcal{T}_{6}\right)+\frac{1}{2}\left[\begin{array}{c}
-\sin \left[1-\lambda\left(\xi_{0}+1\right)\right] \\
-\cos \left[1-\lambda\left(\xi_{0}+1\right)\right]
\end{array}\right]\left(\mathcal{T}_{7}+\mathcal{T}_{4}\right), & 0<\xi_{0}<\frac{1}{2} \\
{\left[\begin{array}{c}
\sin [(\lambda+1)(x-1)] \\
-\cos [(\lambda+1)(x-1)]
\end{array}\right]\left(\mathcal{T}_{5}+\mathcal{T}_{8}+\mathcal{T}_{9}\right)+\left[\begin{array}{c}
-\sin \left[\frac{3}{2}-\lambda-(\lambda+1) x\right] \\
-\cos \left[\frac{3}{2}-\lambda-(\lambda+1) x\right]
\end{array}\right] \mathcal{T}_{10},} & 0<\xi_{0} \leq 1,\end{cases}
$$

where

$$
\begin{aligned}
& \mathcal{T}_{1}=\int_{-1}^{\xi_{0}}\left[\sin [(\lambda-1)(x+1)] f_{1}(x)-\cos [(\lambda-1)(x+1)] f_{2}(x)\right] d x, \\
& \mathcal{T}_{2}=-\int_{\xi_{0}}^{0}\left[\sin \left[\lambda+\frac{1}{2}+(1-\lambda) x\right] f_{1}(x)+\cos \left[\lambda+\frac{1}{2}+(1-\lambda) x\right] f_{2}(x)\right] d x, \\
& \mathcal{T}_{3}=-\frac{1}{2} \int_{0}^{\frac{1}{2}}\left[\sin \left[\lambda+\frac{1}{2}-\lambda x\right] f_{1}(x)+\cos \left[\lambda+\frac{1}{2}-\lambda x\right] f_{2}(x)\right] d x, \\
& \mathcal{T}_{4}=\int_{\frac{1}{2}}^{1}\left[\sin [(\lambda+1)(x-1)] f_{1}(x)-\cos [(\lambda+1)(x-1)] f_{2}(x)\right] d x, \\
& \mathcal{T}_{5}=\int_{-1}^{0}\left[\sin [(\lambda-1)(x+1)] f_{1}(x)-\cos [(\lambda-1)(x+1)] f_{2}(x)\right] d x, \\
& \mathcal{T}_{6}=-\frac{1}{2} \int_{0}^{\xi_{0}}\left[\sin [1-\lambda(x+1)] f_{1}(x)+\cos [1-\lambda(x+1)] f_{2}(x)\right] d x, \\
& \mathcal{T}_{7}=-\int_{\xi_{0}}^{\frac{1}{2}}\left[\sin \left[\lambda+\frac{1}{2}+(1-\lambda) x\right] f_{1}(x)+\cos \left[\lambda+\frac{1}{2}+(1-\lambda) x\right] f_{2}(x)\right] d x, \\
& \mathcal{T}_{8}=-\frac{1}{2} \int_{0}^{\frac{1}{2}}\left[\sin [1-\lambda(x+1)] f_{1}(x)+\cos [1-\lambda(x+1)] f_{2}(x)\right] d x, \\
& \mathcal{T}_{9}=-\int_{\frac{1}{2}}^{\xi_{0}}\left[\sin \left[\frac{3}{2}-\lambda-(\lambda+1) x\right] f_{1}(x)+\cos \left[\frac{3}{2}-\lambda-(\lambda+1) x\right] f_{2}(x)\right] d x, \\
& \mathcal{T}_{10}=\int_{\xi_{0}}^{1}\left[\sin [(\lambda+1)(x-1)] f_{1}(x)-\cos [(\lambda+1)(x-1)] f_{2}(x)\right] d x \text {. }
\end{aligned}
$$

The vector-valued transform (6.12) has the following vector-valued expansion:

$$
\mathfrak{F}(\lambda)=\left[\begin{array}{l}
\mathfrak{F}_{1}(\lambda)=\sum_{n=-\infty}^{\infty} \mathfrak{F}_{1}\left(\frac{1-2 n \pi}{4}\right) \frac{\sin \left[\frac{1}{2}+n \pi-2 \lambda\right]}{\frac{1}{2}-n \pi-2 \lambda} \\
\mathfrak{F}_{2}(\lambda)=\sum_{n=-\infty}^{\infty} \mathfrak{F}_{2}\left(\frac{1-2 n \pi}{4}\right) \frac{\sin \left[\frac{1}{2}+n \pi-2 \lambda\right]}{\frac{1}{2}-n \pi-2 \lambda}
\end{array}\right] .
$$

\section{Competing interests}

The author declares that he has no competing interests.

\section{Acknowledgements}

The author is very grateful to the referees for carefully reading the paper and for their comments and suggestions, which have improved the paper.

Received: 2 August 2015 Accepted: 30 December 2015 Published online: 07 January 2016

\section{References}

1. Levitan, BM, Sargsjan, IS: Introduction to Spectral Theory: Selfadjoint Ordinary Differential Operators. Translations of Mathematical Monographs, vol. 39. Am. Math. Soc., Providence (1975) 
2. Levitan, BM, Sargsjan, IS: Sturm-Liouville and Dirac Operators. Kluwer Academic, Dordrecht (1991)

3. Fulton, CT: Two point boundary value problems with eigenvalue parameter contained in the boundary conditions. Proc. R. Soc. Edinb., Sect. A 77, 293-308 (1977)

4. Tharwat, MM, Yildirim, A, Bhrawy, AH: Sampling of discontinuous Dirac systems. Numer. Funct. Anal. Optim. 34(3), 323-348 (2013)

5. Higgins, JR: Sampling Theory in Fourier and Signal Analysis: Foundations. Oxford University Press, Oxford (1996)

6. Kotel'nikov, V: On the carrying capacity of the 'ether' and wire in telecommunications. In: Material for the First All Union Conference on Questions of Communications, vol. 55, pp. 55-64. Izd. Red. Upr. Svyazi RKKA, Moscow (1933)

7. Shannon, C: Communication in the presence of noise. Proc. IRE 37, 10-21 (1949)

8. Whittaker, E: On the functions which are represented by the expansion of the interpolation theory. Proc. R. Soc. Edinb., Sect. A 35, 181-194 (1915)

9. Zayed, Al: Advances in Shannon's Sampling Theory. CRC Press, Boca Raton (1993)

10. Boas, R: Entire Functions. Academic Press, New York (1954)

11. Paley, R, Wiener, N: Fourier Transforms in the Complex Domain. Am. Math. Soc. Colloquium Publ. Ser., vol. 19. Am. Math. Soc., Providence (1934)

12. Lasser, R: Introduction to Fourier Series. Dekker, New York (1996)

13. Butzer, PL: A survey of the Whittaker-Shannon sampling theorem and some of its extensions. J. Math. Res. Exposition 3, 185-212 (1983)

14. Butzer, P, Higgins, JR, Stens, RL: Sampling theory in signal analysis. In: Pier, JP (ed.) Development of Mathematics 1950-2000, pp. 193-234. Birkhäuser, Basel (2000)

15. Butzer, P, Nasri-Roudsari, G: Kramer's sampling theorem in signal analysis and its role in mathematics. In: Blackledge, JM (ed.) Image Processing: Mathematical Methods and Applications. Proc. IMA Conference, Cranfield University, UK, pp. 49-95. Clarendon, Oxford (1997)

16. Kramer, HP: A generalized sampling theorem. J. Math. Phys. 38, 68-72 (1959)

17. Weiss, P: Sampling theorems associated with Sturm-Liouville systems. Bull. Am. Math. Soc. 163, 242 (1957)

18. Everitt, WN, Garcia, AG, Hernández-Medina, MA: On Lagrange-type interpolation series and analytic Kramer kernels. Results Math. 51, 215-228 (2008)

19. Garcia, AG, Littlejohn, LL: On analytic sampling theory. J. Comput. Appl. Math. 171, 235-246 (2004)

20. Higgins, JR: A sampling principle associated with Saitoh's fundamental theory of linear transformations. In: Saitoh, S, Hayashi, N, Yamamoto, M (eds.) Analytic Extension Formulas and Their Applications. Kluwer Academic, Dordrecht (2001)

21. Abd-alla, MZ, Annaby, MH: Sampling of vector-valued transforms associated with Green's matrix of Dirac systems. J. Math. Anal. Appl. 284, 104-117 (2003)

22. Zayed, A, Garcia, AG: Sampling theorems associated with a Dirac operator and the Hartley transform. J. Math. Anal. Appl. 214, 587-598 (1997)

23. Annaby, MH, Tharwat, MM: On sampling and Dirac systems with eigenparameter in the boundary conditions. J. Appl. Math. Comput. 36, 291-317 (2011)

24. Tharwat, MM: Discontinuous Sturm-Liouville problems and associated sampling theories. Abstr. Appl. Anal. (2011). doi:10.1155/2011/610232

25. Tharwat, MM: On sampling theories and discontinuous Dirac systems with eigenparameter in the boundary conditions. Bound. Value Probl. (2013). doi:10.1186/1687-2770-2013-65

26. Hira, F, Altinisik, N: Dirac system with discontinuities at two points. Abstr. Appl. Anal. 2014, Article ID 927693 (2014)

27. Hira, F, Altinisik, N: Expansion theorems for discontinuous Sturm-Liouville problems. J. Math. Comput. Sci. 4, 335-349 (2014)

28. Coddington, EA, Levinson, N: Theory of Ordinary Differential Equations. McGraw-Hill, New York (1955)

\section{Submit your manuscript to a SpringerOpen ${ }^{\circ}$ journal and benefit from:}

- Convenient online submission

Rigorous peer review

- Immediate publication on acceptance

- Open access: articles freely available online

- High visibility within the field

- Retaining the copyright to your article 